\title{
Pre-aggregation functions: construction and an application
}

\author{
Giancarlo Lucca, José Antonio Sanz, Graçaliz Pereira Dimuro, Benjamín Bedregal, Radko Mesiar, Anna \\ Kolesárová, Humberto Bustince, IEEE Senior Member
}

\begin{abstract}
In this work we introduce the notion of preaggregation function. Such a function satisfies the same boundary conditions as an aggregation function, but, instead of requiring monotonicity, only monotonicity along some fixed direction (directional monotonicity) is required. We present some examples of such functions. We propose three different methods to build pre-aggregation functions. We experimentally show that in fuzzy rule-based classification systems, when we use one of these methods, namely, the one based on the use of the Choquet integral replacing the product by other aggregation functions, if we consider the minimum or the Hamacher product t-norms for such construction, we improve the results obtained when applying the fuzzy reasoning methods obtained using two classical averaging operators like the maximum and the Choquet integral.
\end{abstract}

Index Terms-Aggregation functions, directional monotonicity, fuzzy measures, Choquet integral, fuzzy rule-based classification systems, fuzzy reasoning method

\section{INTRODUCTION}

Aggregation functions [1], [2] are crucial tools nowadays to deal with many computation problems [3], [4], [5], [6], [7]. The key property for defining them, apart from the boundary conditions, is monotonicity and, more specifically, monotone increasingness. However, some other statistical tools, such as the mode, are not included in this family, although they are useful, since, even if they are properly defined as functions, monotonicity is violated.

The problem of relaxing the definition of monotonicity has recently attracted a lot of interest. In [8], Wilkin and Beliakov proposed the notion of weak monotonicity in order to extend the usual monotonicity property. In this case, monotonicity is

This work was supported in part by the Spanish Ministry of Science and Technology under projects TIN2008-06681-C06-01, TIN201015055, TIN2013-40765-P, TIN2011-29520, by the Brazilian funding agency CNPQ under Processes 481283/2013-7, 306970/2013-9, 232827/2014-1 and $307681 / 2012-2$, and by grants VEGA 1/0419/13 and VEGA 1/0420/15.

G. Lucca and G. Pereira Dimuro are with PPGCOMP, Centro de Ciencias Computacionais, Universidade Federal do Rio Grande, Rio Grande, Brazil, e-mail: gracalizdimuro@furg.br

J. Sanz and H. Bustince are with the Departamento of Automática y Computación and with the Institute of Smart Cities, Universidad Publica de Navarra, Navarra, 31006 Spain e-mails: \{joseantonio.sanz,bustince\}@unavarra.es.

B. Bedregal is with Departamento de Informática e Matemática Aplicada, Universidade Federal do Rio Grande do Norte, Natal, Brazil, e-mail: bedregal@dimap.ufrn.br.

R. Mesiar is with the Slovak University of Technology, Radlinskeho 11, 81005 Bratislava, Slovakia and with the Institute of Information Theory and Automation, Academy of Sciences of the Czech Republic, 18208 Prague, Czech Republic

A. Kolesárová is with the Institute of Information Engineering, Automation and Mathematics, Slovak University of Technology, 81237 Bratislava, Slovakia. required only along the direction of the first quadrant diagonal. This concept of weak monotonicity has been further extended by Bustince et al. [9] by introducing the notion of directional monotonicity, which allows monotonicity along (some) fixed ray. In particular, directionally monotone functions encompass weak monotone functions, as well as the mode and any aggregation function.

In particular, in this paper we consider the following objectives:

1) To introduce the concept of pre-aggregation functions.

2) To study the first properties of these new functions.

3) To introduce three different methods for building preaggregation functions.

4) To show an application where the introduction of the new concept of pre-aggregation function is justified.

To achieve these goals we use the notion of directional monotonicity. Moreover, for one of the construction methods that we propose, in the definition of the Choquet integral we replace the product by the minimum or the Hamacher product t-norm, and, in this way, we obtain pre-aggregation functions that are not aggregation functions. We show that using these new functions in a Fuzzy Rule-based Classification System (FRBCS), and, in particular, in the Fuzzy Reasoning Method (FRM) of FARC-HD [10], which is currently one of the most accurate FRBCSs, the obtained results are better than both applying the classical Choquet integral and the well-known FRM of the winning rule.

This paper is organized as follows. In Section II, we present some related preliminary concepts that are necessary to understand the paper. In Section III we introduce the notion of pre-aggregation function and discuss some properties. Three methods of construction of pre-aggregation functions are described in Section IV. The generalization of the FRM using pre-aggregation functions is described in detail in Section V. The experimental framework and the analysis of the obtained results when considering some of our pre-aggregation functions are reported in Section VI. In Section VII we draw the main conclusions and the detailed results of the experiments are available in the Appendix.

\section{PRELIMINARIES}

\section{A. Aggregation functions}

An important class of operators that are used in this paper are the aggregation functions [1], [11]:

Definition 2.1: A function $A:[0,1]^{n} \rightarrow[0,1]$ is said to be an $n$-ary aggregation function if the following conditions hold: 
TABLE I: T-norms used in this paper

\begin{tabular}{|c|c|}
\hline Name & Definition \\
\hline Minimum & $T_{M}(x, y)=\min \{x, y\}$ \\
\hline Algebraic Product & $T_{P}(x, y)=x y$ \\
\hline Łukasiewicz & $T_{€}(x, y)=\max \{0, x+y-1\}$ \\
\hline Drastic Product & $T_{D P}(x, y)= \begin{cases}x & \text { if } y=1 \\
y & \text { if } x=1 \\
0 & \text { otherwise }\end{cases}$ \\
\hline Nilpotent Minimum & $T_{N M}(x, y)= \begin{cases}\min \{x, y\} & \text { if } x+y>1 \\
0 & \text { otherwise }\end{cases}$ \\
\hline Hamacher Product & $T_{H P}(x, y)= \begin{cases}0 & \text { if } x=y=0 \\
\frac{x y}{x+y-x y} & \text { otherwise }\end{cases}$ \\
\hline
\end{tabular}

(A1) $A$ is increasing ${ }^{1}$ in each argument: for each $i \in$ $\{1, \ldots, n\}$, if $x_{i} \leq y$, then $A\left(x_{1}, \ldots, x_{n}\right) \leq$ $A\left(x_{1}, \ldots, x_{i-1}, y, x_{i+1}, \ldots, x_{n}\right)$;

(A2) $A$ satisfies the boundary conditions: $A(0, \ldots, 0)=0$ and $A(1, \ldots, 1)=1$.

Definition 2.2: A bivariate aggregation function $T$ : $[0,1]^{2} \rightarrow[0,1]$ is a t-norm if, for all $x, y, z \in[0,1]$, it satisfies the following properties:

(T1) Commutativity: $T(x, y)=T(y, x)$;

(T2) Associativity: $T(x, T(y, z))=T(T(x, y), z)$;

(T3) Boundary condition: $T(x, 1)=x$.

If $T$ satisfies (T3) (and also $T(1, x)=x$ ) only, then it is called a semi-copula.

Since t-norms are associative, it is possible to extend each t-norm $T$ in a unique way to an $n$-ary operation in the usual way by induction [12]. The bivariate t-norms that are used in this paper are presented in Table I. Observe that a convex combination of t-norms is a (commutative) semicopula, but not a t-norm, in general, since associativity may be violated.

\section{B. Fuzzy measures}

In this subsection, we recall the notion of fuzzy measure, which is going to be a key tool for constructing some of our examples of pre-aggregation functions.

In the following, consider the set $N=\{1, \ldots, n\}$ for an arbitrary positive integer $n$.

Definition 2.3: A function $\mathfrak{m}: 2^{N} \rightarrow[0,1]$ is a fuzzy measure if, for all $X, Y \subseteq N$, it satisfies the following properties:

( $\mathfrak{m} 1)$ Increasingness: if $X \subseteq Y$, then $\mathfrak{m}(X) \leq \mathfrak{m}(Y)$;

$(\mathfrak{m} 2)$ Boundary conditions: $\mathfrak{m}(\emptyset)=0$ and $\mathfrak{m}(N)=1$.

In the context of aggregation functions, fuzzy measures are used for evaluating the relationship among the elements to be aggregated, which represents the importance of a coalition. The fuzzy measures considered in this paper, defined for $A \subseteq$ $N$, are the following:

Uniform measure:

$$
\mathfrak{m}_{U}(A)=\frac{|A|}{n} .
$$

${ }^{1}$ In this paper, an increasing (decreasing) function does not need to be strictly increasing (decreasing).
Dirac's measure: For a previously fixed $i \in N$,

$$
\mathfrak{m}_{D}^{i}(A)= \begin{cases}1 & \text { if } i \in A \\ 0 & \text { if } i \notin A .\end{cases}
$$

Additive measure (Wmean): Take $W=$ $\left(w_{1}, \ldots, w_{n}\right) \in[0,1]^{n}$ such that $\sum_{i=1}^{n} w_{i}=1$. Consider

$$
\mathfrak{m}_{W}(\{i\})=w_{i}
$$

Then, for $|A|>1$, define:

$$
\mathfrak{m}_{W}(A)=\sum_{i \in A} w_{i}
$$

Symmetric measure (OWA): Take $W=$ $\left(w_{1}, \ldots, w_{n}\right) \in[0,1]^{n}$ such that $\sum_{i=1}^{n} w_{i}=1$. Then, for any non-empty subset $A$, define:

$$
\mathfrak{m}_{s W}(A)=\sum_{i=1}^{|A|} w_{i} .
$$

Note that this expression is different from Eq. (3) since in this case only the cardinal of each subset $A$ is taken into account.

Power measure:

$$
\mathfrak{m}_{P M}(A)=\left(\frac{|A|}{n}\right)^{q}, \text { with } q>0 .
$$

Observe also that from the considered fuzzy measures, $\mathfrak{m}_{U}, \mathfrak{m}_{D}^{i}$ and $\mathfrak{m}_{W}$ are additive and $\mathfrak{m}_{U}, \mathfrak{m}_{s W}$ and $\mathfrak{m}_{P M}$ are symmetric, that is, the measure of any subset $A$ only depends on the cardinality of $A$.

The Choquet integral generalizes the Lebesgue integral, which is defined with respect to additive measures. However, the Choquet integral is defined with respect to fuzzy measures. In this paper, we consider only the discrete Choquet integral, related to fuzzy measures, which are defined on finite spaces:

Definition 2.4: [1, Definition 1.74] Let $\mathfrak{m}: 2^{N} \rightarrow[0,1]$ be a fuzzy measure. The discrete Choquet integral of $\mathbf{x}=$ $\left(x_{1}, \ldots, x_{n}\right) \in[0,1]^{n}$ with respect to $\mathfrak{m}$ is defined as a function $C_{\mathfrak{m}}:[0,1]^{n} \rightarrow[0,1]$, given by

$$
C_{\mathfrak{m}}(\mathbf{x})=\sum_{i=1}^{n}\left(x_{(i)}-x_{(i-1)}\right) \cdot \mathfrak{m}\left(A_{(i)}\right),
$$

where $\left(x_{(1)}, \ldots, x_{(n)}\right)$ is an increasing permutation on the input $\mathbf{x}$, that is, $0 \leq x_{(1)} \leq \ldots \leq x_{(n)}$, with the convention that $x_{(0)}=0$, and $A_{(i)}=\{(i), \ldots,(n)\}$ is the subset of indices of $n-i+1$ largest components of $\mathbf{x}$.

The Choquet integral combines the inputs in such a way that the importance of the different groups of inputs (coalitions) may be taken into account. Allowing to assign importance to all possible groups of criteria, the Choquet integral offers greater flexibility in the aggregation modelling. Since the weighted arithmetic mean and OWA operators are special cases of the Choquet integral, with respect to additive and symmetric fuzzy measures, respectively, Choquet integralbased aggregation functions represent a larger class of aggregation functions [1], [13], [14].

Note that the Choquet integral with respect to $\mathfrak{m}_{W}$ is a 
weighted arithmetic mean, and with respect to $\mathfrak{m}_{s W}$ is an OWA operator $^{2}$. These facts explain the acronyms we have chosen in the present work for these measures.

\section{Directional monotonicity}

This subsection is devoted to recalling the basic concept for our definition of pre-aggregation function, that of directional monotonicity [9].

Definition 2.5: Let $\vec{r}=\left(r_{1}, \ldots, r_{n}\right)$ be a real $n$ dimensional vector, $\vec{r} \neq \overrightarrow{0}$. A function $F:[0,1]^{n} \rightarrow[0,1]$ is $\vec{r}$-increasing if for all points $\left(x_{1}, \ldots, x_{n}\right) \in[0,1]^{n}$ and for all $c>0$ such that $\left(x_{1}+c r_{1}, \ldots, x_{n}+c r_{n}\right) \in[0,1]^{n}$ it holds

$$
F\left(x_{1}+c r_{1}, \ldots, x_{n}+c r_{n}\right) \geq F\left(x_{1}, \ldots, x_{n}\right) .
$$

That is, an $\vec{r}$-increasing function is a function which is increasing along the ray (direction) determined by the vector $\vec{r}$. For this reason, we say that $F$ is directionally monotone, or, more specifically, directionally increasing. Note that every increasing function (in the usual sense) is, in particular, $\vec{r}$ increasing, for every non-negative real vector $\vec{r}$. However, the class of directionally increasing functions is much wider than that of aggregation functions. For instance:

- Fuzzy implication functions (see [21]) are $(-1,1)$ increasing functions. This implies that many other functions, which are widely used in applications and which can be obtained from implication functions, are also directionally increasing. This is the case, for instance, of some subsethood measures (see [22]);

- Many functions used for comparison of data are also directionally increasing. In particular, this is the case of those based on component-wise comparison by means of the Euclidean distance $|x-y|$, as for restricted equivalence functions [23];

- Weakly increasing functions ([8]) are a particular case of directionally increasing functions, with $\vec{r}=(1, \ldots, 1)$.

\section{Pre-Aggregation FUNCTIONS}

In this section we introduce the notion of pre-aggregation function and discuss some properties and construction methods.

Definition 3.1: A function $F:[0,1]^{n} \rightarrow[0,1]$ is said to be an $n$-ary pre-aggregation function if the following conditions hold:

(PA1) There exists a real vector $\vec{r} \in[0,1]^{n}(\vec{r} \neq \overrightarrow{0})$ such that $F$ is $\vec{r}$-increasing.

(PA2) $F$ satisfies the boundary conditions: $F(0, \ldots, 0)=0$ and $F(1, \ldots, 1)=1$.

Example 3.1: Some examples of pre-aggregation functions are the following.

(i) Consider the mode, $\operatorname{Mod}\left(x_{1}, \ldots, x_{n}\right)$, defined as the function that gives back the value which appears most times in the considered $n$-tuple, or the smallest of the values that appears most times, in case there is more than

\footnotetext{
${ }^{2}$ The OWA operators were first introduced by Yager [15], and several forms and usage of OWA operators have been discussed in the literature [16], [17], [18], [19], [20].
}

one. Then, the mode is $(1, \ldots, 1)$-increasing, and it is a particular case of pre-aggregation function which is not an aggregation function.

(ii) $F(x, y)=x-(\max \{0, x-y\})^{2}$ is, for instance, $(0,1)$ increasing, and it is an example of a pre-aggregation function which is not an aggregation function.

(iii) Weakly increasing functions satisfying the boundary conditions (PA2) are also pre-aggregation functions which need not be aggregation functions.

(iv) Take $\lambda \in] 0,1\left[\right.$. The weighted Lehmer mean $L_{\lambda}$ : $[0,1]^{2} \rightarrow[0,1]$, given by

$$
L_{\lambda}(x, y)=\frac{\lambda x^{2}+(1-\lambda) y^{2}}{\lambda x+(1-\lambda) y}
$$

(with convention $0 / 0=0)$ is $(1-\lambda, \lambda)$-increasing, so it is a pre-aggregation function.

(v) Define $A, B:[0,1]^{2} \rightarrow[0,1]$ by

$$
A(x, y)= \begin{cases}x(1-x) & \text { if } y \leq 3 / 4 \\ 1 & \text { otherwise }\end{cases}
$$

and

$$
B(x, y)= \begin{cases}y(1-y) & \text { if } x \leq 3 / 4 \\ 1 & \text { otherwise }\end{cases}
$$

Then both $A$ and $B$ are pre-aggregation functions which are not aggregation functions. In fact, $A$ is $(0, a)$ increasing for any $a>0$ but for no other direction $\vec{r}=(a, b), b>0$, while $B$ is $(b, 0)$-increasing for any $b>0$ but for no other direction $\vec{r}=(a, b), a>0$. However, $C=(A+B) / 2$ is not a pre-aggregation function, just illustrating the fact that the class of all preaggregation functions with a fixed dimension $n$ is not a convex class.

If $F$ is a pre-aggregation function with respect to a vector $\vec{r}$ we just say that $F$ is an $\vec{r}$-pre-aggregation function.

Remark 3.1: Note that if $A:[0,1]^{n} \rightarrow[0,1]$ is an aggregation function, then $A$ is also a pre-aggregation function. In fact, if, for a non-zero vector $\vec{r} \in[0,1]^{n}$ we denote by $P A_{\vec{r}}$ the class of all $\vec{r}$-increasing pre-aggregation functions, then the class of all pre-aggregation functions $P A$ is the union of all these classes $P A_{\vec{r}}$, while the class of all aggregation functions is the intersection of all the classes $P A_{\vec{r}}$. The latter intersection is the same as the intersection over $P A_{\overrightarrow{e_{i}}}$, where $\overrightarrow{e_{i}}=(0, . .1, . .0), i \in\{1, \ldots, n\}$, is the vector having 1 as $i$-th value, and all other coordinates are equal to zero.

Note that the reverse of the first claim of Remark 3.1 does not hold, as the cases considered in Example 3.1 (i) and (ii) show. Pre-aggregation functions which are not aggregation functions will be called proper pre-aggregation functions. However, we can use aggregation functions to obtain directionally increasing functions as follows.

The next results were proved for directionally monotone functions in our recent paper [9].

Proposition 3.1: Let $A:[0,1]^{m} \rightarrow[0,1]$ be an aggregation function. Let $F_{i}:[0,1]^{n} \rightarrow[0,1](i \in\{1, \ldots, m\})$ be a family of $m \vec{r}$-pre-aggregation functions for the same vector $\vec{r} \in[0,1]^{n}$. Then, the function $A\left(F_{1}, \ldots, F_{m}\right):[0,1]^{n} \rightarrow$ 
$[0,1]$, defined as

$$
\begin{gathered}
A\left(F_{1}, \ldots, F_{m}\right)\left(x_{1}, \ldots, x_{n}\right)= \\
A\left(F_{1}\left(x_{1}, \ldots, x_{n}\right), \ldots, F_{m}\left(x_{1}, \ldots, x_{n}\right)\right)
\end{gathered}
$$

is also an $\vec{r}$-pre-aggregation function.

Proof:

Due to ([9], Proposition 3), only the boundary conditions for the functions $\left(F_{1}, \ldots, F_{m}\right)$ should be guaranteed. However, their validity is obvious.

The following corollary is straightforward.

Corollary 3.1: Let $F_{1}, F_{2}:[0,1]^{n} \rightarrow[0,1]$ be two $\vec{r}$-preaggregation functions for the same vector $\vec{r} \in[0,1]^{n}$. Then:

(i) $\frac{F_{1}+F_{2}}{2}$ is also an $\vec{r}$-pre-aggregation function.

(ii) $F_{1} F_{2}$ is also an $\vec{r}$-pre-aggregation function.

Regarding duality, we can state the following.

Proposition 3.2: Let $F:[0,1]^{n} \rightarrow[0,1]$ be an $\vec{r}$-preaggregation function for $\vec{r} \in[0,1]^{n}$. Then, the function

$$
F^{d}\left(x_{1}, \ldots, x_{n}\right)=1-F\left(1-x_{1}, \ldots, 1-x_{n}\right)
$$

is also an $\vec{r}$-pre-aggregation function.

Proof:

Obviously, $F^{d}(0, \ldots, 0)=0$ and $F^{d}(1, \ldots, 1)=1$. Now, the result follows from ([9], Proposition 3).

The following corollary is now straight.

Corollary 3.2: Let $F$ be an $\vec{r}$ - pre-aggregation function. Then, the function $\frac{F+F^{d}}{2}$ is a self-dual $\vec{r}$-pre-aggregation function.

\section{THREE METHODS OF CONSTRUCTING PRE-AGGREGATION FUNCTIONS}

In this section we introduce and illustrate three methods of constructing pre-aggregation functions. The first method is based on the composition of appropriate functions, the second one is inspired by the construction of the discrete Choquet integral, and the third of the proposed methods is inspired by the construction of the discrete Sugeno integral.

\section{A. Construction of pre-aggregation functions by composition}

Fix $n \in \mathbb{N}$. Let $I$ be a proper subset of $N=\{1, \ldots, n\}$ and consider that $I=\left\{i_{1}, \ldots, i_{k}\right\}$ with $i_{1}<\ldots<i_{k}$. For an $n$ tuple $\mathbf{x}=\left(x_{1}, \ldots, x_{n}\right) \in[0,1]^{n}$, its $I$-projection is a $k$-tuple $\mathbf{x}_{I}=\left(x_{i_{1}}, \ldots, x_{i_{k}}\right)$, where $k$ is the cardinality of $I$. We will use $I$-projections $\mathbf{x}_{I}$ of points $\mathbf{x} \in[0,1]^{n}$ and $I$-projections $\vec{r}_{I}$ of (geometrical) vectors $\vec{r} \in[0,1]^{n}$ as well. Finally, for a function $F:[0,1]^{n} \rightarrow[0,1]$, let $\mathcal{D}^{\uparrow}(F)=\left\{\vec{r} \in[0,1]^{n}\right.$ $F$ is $\vec{r}$ - increasing . Note that the zero vector is not excluded now.

Proposition 4.1: Let $\left\{I_{1}, \ldots, I_{k}\right\}$ be a partition of $N, k>$ 1. For $j \in\{1, \ldots, k\}$, let $n_{j}=\left|I_{j}\right|$ and consider functions $F_{j}:[0,1]^{n_{j}} \rightarrow[0,1]$ such that $F_{j}(1, \ldots, 1)=1$. Then, for any aggregation function $G:[0,1]^{k} \rightarrow[0,1]$, the composite function $H:[0,1]^{n} \rightarrow[0,1]$ defined by

$$
H(\mathbf{x})=G\left(F_{1}\left(\mathbf{x}_{I_{1}}\right), \ldots, F_{k}\left(\mathbf{x}_{I_{k}}\right)\right)
$$

is $\vec{r}$-increasing for any vector $\vec{r} \in[0,1]^{n}$ such that $\vec{r}_{I_{j}} \in$ $\mathcal{D}^{\uparrow}\left(F_{j}\right), j=1, \ldots, k$, and $H(\mathbf{1})=1$. Moreover, if there is a $j_{0} \in\{1, \ldots, k\}$ such that $F_{j_{0}}$ is a pre-aggregation function, and 0 is an annihilator of $G$, then the function $H$ is a preaggregation function.

Proof: Clearly, $H(\mathbf{1})=G\left(F_{1}\left(\mathbf{1}_{I_{1}}\right), \ldots, F_{k}\left(\mathbf{1}_{I_{k}}\right)\right)=$ $G(1, \ldots, 1)=1$. Moreover, if $F_{j_{0}}(0, \ldots, 0)=0$ for some $j_{0} \in\{1, \ldots, k\}$ and 0 is an annihilator of $G$, then

$$
\begin{aligned}
H(\mathbf{0}) & =G\left(F_{1}\left(\mathbf{0}_{I_{1}}\right), \ldots, F_{j_{0}}\left(\mathbf{0}_{I_{j_{0}}}\right), \ldots, F_{k}\left(\mathbf{0}_{I_{k}}\right)\right) \\
& =G\left(F_{1}\left(\mathbf{0}_{I_{1}}\right), \ldots, 0, \ldots, F_{k}\left(\mathbf{0}_{I_{k}}\right)\right)=0 .
\end{aligned}
$$

Next, consider a vector $\vec{r} \in[0,1]^{n}$ such that $\vec{r}_{I_{j}} \in \mathcal{D}^{\uparrow}\left(F_{j}\right)$ for each $j=1, \ldots, k$. Then, for any $c>0$ and $\mathbf{x} \in[0,1]^{n}$ such that also $\mathrm{x}+c \vec{r} \in[0,1]^{n}$, it holds that

$$
\begin{aligned}
H(\mathbf{x}+c \vec{r}) & =G\left(F_{1}\left(\mathbf{x}_{I_{1}}+c \vec{r}_{I_{1}}\right), \ldots, F_{k}\left(\mathbf{x}_{I_{k}}+c \vec{r}_{I_{k}}\right)\right) \\
& \geq G\left(F_{1}\left(\mathbf{x}_{I_{1}}\right), \ldots, F_{k}\left(\mathbf{x}_{I_{k}}\right)\right)=H(\mathbf{x}),
\end{aligned}
$$

where the inequality follows from the increasing monotonicity of the aggregation function $G$, and the fact that $F_{j}\left(\mathbf{x}_{I_{j}}+c \vec{r}_{I_{j}}\right) \geq F_{j}\left(\mathbf{x}_{I_{j}}\right), j=1, \ldots, k$.

Now, suppose that $F_{j_{0}}$ is a pre-aggregation function, i.e., $F_{j_{0}}(0, \ldots, 0)=0$ and $F_{j_{0}}$ is $\vec{v}$-increasing for some non-zero vector $\vec{v} \in[0,1]^{n_{j_{0}}}$. Due to the above mentioned facts, $H$ satisfies the boundary conditions and is directionally increasing in the direction of a non-zero vector $\vec{r} \in[0,1]^{n}$ such that $\vec{r}_{I_{j_{0}}}=\vec{v}$ and $\vec{r}_{N \backslash I_{j_{0}}}=$ $(0, \ldots, 0)$, which proves that $H$ is a pre-aggregation function.

Example 4.1: Let $n=2$ and $\left.\left.\vec{v}=\left(v_{1}, v_{2}\right) \in\right] 0,1\right]^{2}$. For obtaining a proper pre-aggregation function which is $\vec{v}$ increasing, it is enough to consider the weighted Lehmer mean $L_{\lambda}:[0,1]^{2} \rightarrow[0,1]$ with $\lambda=\frac{v_{2}}{v_{1}+v_{2}}$, see Example 3.1(iv), given by

$$
L_{\lambda}(x, y)=\frac{v_{2} x^{2}+v_{1} y^{2}}{v_{2} x+v_{1} y} .
$$

This fact and Proposition 4.1 allow us to construct a preaggregation function $H$ which is directionally increasing in the direction of any a-priori given vector $\overrightarrow{0} \neq \vec{r} \in[0,1]^{n}$.

Consider, for example, $n=4$ and $\vec{r}=(0.5,0.4,0.3,0.7)$. Let $G=T_{M}, I_{1}=\{1,3\}, I_{2}=\{2,4\}, F_{1}=L_{3 / 8}, F_{2}=$ $L_{7 / 11}$. Then $H:[0,1]^{4} \rightarrow[0,1]$ given by

$$
H\left(x_{1}, x_{2}, x_{3}, x_{4}\right)=\min \left\{\frac{3 x_{1}^{2}+5 x_{3}^{2}}{3 x_{1}+5 x_{3}}, \frac{7 x_{2}^{2}+4 x_{4}^{2}}{7 x_{2}+4 x_{4}}\right\}
$$

is an $\vec{r}$-increasing proper pre-aggregation function.

\section{B. Choquet-like construction method of pre-aggregation func-} tions

This method is inspired in the way the Choquet integral is built, replacing the product operation in Equation (6) by other aggregation functions.

Let $\mathfrak{m}: 2^{N} \rightarrow[0,1]$ be a fuzzy measure and $M:[0,1]^{2} \rightarrow$ $[0,1]$ be a function such that $M(0, x)=0$ for every $x \in[0,1]$. Taking as basis the Choquet integral, we define the function 


$$
\begin{aligned}
C_{\mathfrak{m}}^{M}:[0,1]^{n} \rightarrow[0, n] \text { by } & \\
C_{\mathfrak{m}}^{M}(\mathbf{x}) & =\sum_{i=1}^{n} M\left(x_{(i)}-x_{(i-1)}, \mathfrak{m}\left(A_{(i)}\right)\right),
\end{aligned}
$$

where $N=\{1, \ldots, n\},\left(x_{(1)}, \ldots, x_{(n)}\right)$ is an increasing permutation on the input $\mathbf{x}$, that is, $0 \leq x_{(1)} \leq \ldots \leq x_{(n)}$, with the convention that $x_{(0)}=0$, and $A_{(i)}=\{(i), \ldots,(n)\}$ is the subset of indices of $n-i+1$ largest components of $\mathbf{x}$. Note that $C_{\mathrm{m}}^{M}$ is well defined by (7) even if the permutation is not unique.

Now we have the following result.

Theorem 4.1: Let $M:[0,1]^{2} \rightarrow[0,1]$ be a function such that for all $x, y \in[0,1]$ it satisfies $M(x, y) \leq x, M(x, 1)=$ $x, M(0, y)=0$ and $M$ is (1,0)-increasing. Then, for any fuzzy measure $\mathfrak{m}, C_{\mathfrak{m}}^{M}$ is a pre-aggregation function which is idempotent and averaging, i.e.,

$$
\min \left(x_{1}, \ldots, x_{n}\right) \leq C_{\mathfrak{m}}^{M}\left(x_{1}, \ldots, x_{n}\right) \leq \max \left(x_{1}, \ldots, x_{n}\right) .
$$

Proof: Note that

$$
\begin{aligned}
C_{\mathfrak{m}}^{M}\left(x_{1}, \ldots, x_{n}\right) & =\sum_{i=1}^{n} M\left(x_{(i)}-x_{(i-1)}, \mathfrak{m}\left(A_{(i)}\right)\right) \\
& \leq \sum_{i=1}^{n}\left(x_{(i)}-x_{(i-1)}\right) \\
& =x_{(n)}=\max \left(x_{1}, \ldots, x_{n}\right)
\end{aligned}
$$

From these two inequalities, idempotency follows. Besides

$$
\begin{aligned}
\min \left(x_{1}, \ldots, x_{n}\right) & =x_{(1)}=M\left(x_{(1)}-x_{(0)}, \mathfrak{m}\left(A_{(1)}\right)\right) \\
& \leq C_{\mathfrak{m}}^{M}\left(x_{1}, \ldots, x_{n}\right)
\end{aligned}
$$

Finally, take $\vec{r}=\overrightarrow{1}=(1, \ldots, 1)$. Note that in Equation (7), for $i \geq 2$, it follows that, for any $c>0$

$$
\begin{gathered}
M\left(x_{(i)}+c-\left(x_{(i-1)}+c\right), \mathfrak{m}\left(A_{(i)}\right)\right) \\
=M\left(x_{(i)}-x_{(i-1)}, \mathfrak{m}\left(A_{(i)}\right)\right)
\end{gathered}
$$

whereas, for $i=1$

$$
\begin{gathered}
M\left(x_{(1)}+c-x_{(0)}, \mathfrak{m}\left(A_{(1)}\right)\right)=M\left(x_{(1)}+c, \mathfrak{m}\left(A_{(1)}\right)\right) \\
\geq M\left(x_{(1)}, \mathfrak{m}\left(A_{(1)}\right)\right)
\end{gathered}
$$

so $C_{\mathfrak{m}}^{M}$ is $\overrightarrow{1}$-increasing.

Remark 4.1: Under the constraints of Theorem 4.1, we cannot ensure the monotonicity of $C_{\mathfrak{m}}^{M}$, i.e., $C_{\mathfrak{m}}^{M}$ is, in general, a proper pre-aggregation function. To see it, observe the following:

(i) Take $M(x, y)=T_{M}(x, y)$. Consider $N=\{1,2,3,4\}$ and the uniform measure $\mathfrak{m}=\mathfrak{m}_{U}$ given in Equation (1). Then, we have that

$$
\begin{gathered}
C_{\mathfrak{m}}^{T_{M}}(0.05,0.1,0.7,0.9)=0.8, \quad \text { whereas } \\
C_{\mathfrak{m}}^{T_{M}}(0.05,0.1,0.8,0.9)=0.7
\end{gathered}
$$

so $C_{\mathfrak{m}}^{T_{M}}$ is not an increasing function and hence it is not an aggregation function.

(ii) Consider the Łukasiewicz t-norm $T_{\succeq}(x, y)=\max \{0, x+$ $y-1\}$. Again, for $N=\{1,2,3,4\}$ and the uniform measure $\mathfrak{m}=\mathfrak{m}_{U}$ we have that

$$
\begin{aligned}
& C_{\mathfrak{m}}^{T_{\mathfrak{E}}}(0.05,0.1,0.7,0.9)=0.15 \text {, whereas } \\
& C_{\mathfrak{m}}^{T} \mathfrak{t}^{T}(0.05,0.2,0.7,0.9)=0.05,
\end{aligned}
$$

so $C_{\mathfrak{m}}^{T} \succeq$ is not an increasing function and hence it is not an aggregation function. Analogous counterexamples can be found for the cases of the drastic product, the Hamacher product or the nilpotent minimum t-norms.

Consider $N=\{1, \ldots, n\}$ and a fuzzy measure $\mathfrak{m}: 2^{N} \rightarrow$ $[0,1]$. In Table II, we present the value of $C_{\mathfrak{m}}^{T}$, which are preaggregation functions but not aggregation functions, for the different t-norms given in Table I.

\section{Sugeno-like construction method of pre-aggregation func-} tions

In this subsection we follow the notation of Definition 2.4. Recall that the formula for the discrete Sugeno integral $S_{\mathfrak{m}}:[0,1]^{n} \rightarrow[0,1]$ can be written as

$$
S_{\mathfrak{m}}(\mathbf{x})=\bigvee_{i=1}^{n} \min \left\{x_{(i)}, \mathfrak{m}\left(A_{(i)}\right)\right\}
$$

Inspired by this formula, for any function $M:[0,1]^{2} \rightarrow[0,1]$, we define the function $S_{\mathfrak{m}}^{M}:[0,1]^{n} \rightarrow[0,1]$ by the formula

$$
S_{\mathfrak{m}}^{M}(\mathbf{x})=\bigvee_{i=1}^{n} M\left(x_{(i)}, \mathfrak{m}\left(A_{(i)}\right)\right) .
$$

We prove a sufficient condition for $M$ ensuring that $S_{\mathfrak{m}}^{M}$ is a pre-aggregation function for any fuzzy measure $\mathfrak{m}$.

Proposition 4.2: Let $M:[0,1]^{2} \rightarrow[0,1]$ be a function increasing in the first variable and let for each $y \in[0,1]$, $M(0, y)=0$ and $M(1,1)=1$. Then $S_{\mathfrak{m}}^{M}$ defined in (8) is a pre-aggregation function for any fuzzy measure $\mathfrak{m}$.

Proof: It is easy to check that, for any $\mathfrak{m}$,

$$
S_{\mathfrak{m}}^{M}(\mathbf{0})=\bigvee_{i=1}^{n} M\left(0, \mathfrak{m}\left(A_{(i)}\right)\right)=0
$$

and

$S_{\mathfrak{m}}^{M}(\mathbf{1})=\bigvee_{i=1}^{n} M\left(1, \mathfrak{m}\left(A_{(i)}\right)\right)=M\left(1, \mathfrak{m}\left(A_{(1)}\right)\right)=M(1,1)=1$.

Moreover, for vector $\overrightarrow{1}=(1, \ldots, 1)$ we get

$$
\begin{gathered}
S_{\mathfrak{m}}^{M}(\mathbf{x}+c \overrightarrow{1})=\bigvee_{i=1}^{n} M\left(x_{(i)}+c, \mathfrak{m}\left(A_{(i)}\right)\right) \\
\geq \bigvee_{i=1}^{n} M\left(x_{(i)}, \mathfrak{m}\left(A_{(i)}\right)\right)=S_{\mathfrak{m}}^{M}(\mathbf{x}),
\end{gathered}
$$

i.e., $S_{\mathfrak{m}}^{M}$ is $\overrightarrow{1}$-increasing, which completes the proof that $S_{\mathfrak{m}}^{M}$ is a pre-aggregation function.

Note that any function $M$ satisfying the constraints of Proposition 4.2 is, in fact, a binary $(1,0)$-increasing pre- 
TABLE II: Some pre-aggregation functions obtained using the t-norms

\begin{tabular}{ll}
\hline T-Norm & Resulting pre-aggregation function \\
\hline Minimum & $C_{\mathfrak{m}}^{T_{M}}(\mathbf{x})=\sum_{i=1}^{n} \min \left\{x_{(i)}-x_{(i-1)}, \mathfrak{m}\left(A_{(i)}\right)\right\}$ \\
Eukasiewicz & $C_{\mathfrak{m}}^{T_{\mathfrak{E}}}(\mathbf{x})=\sum_{i=1}^{n} \max \left\{0, x_{(i)}-x_{(i-1)}+\mathfrak{m}\left(A_{(i)}\right)-1\right\}$ \\
Drastic Product & $C_{\mathfrak{m}}^{D P}(\mathbf{x})=\sum_{i=1}^{n} \begin{cases}x_{(1)} & \text { if } i=1 \\
\mathfrak{m}\left(A_{(i)}\right) & \text { if } x_{(i)}-x_{(i-1)}=1 \\
0 & \text { otherwise }\end{cases}$ \\
Nilpotent Minimum & $C_{\mathfrak{m}}^{N M}(\mathbf{x})=\sum_{i=1}^{n}\left\{\begin{array}{l}\min \left\{x_{(i)}-x_{(i-1)}, \mathfrak{m}\left(A_{(i)}\right)\right\} \\
\text { if } x_{(i)}-x_{(i-1)}+\mathfrak{m}\left(A_{(i)}\right)>1 \\
\text { otherwise }\end{array}\right.$ \\
Hamacher Product & $C_{\mathfrak{m}}^{H} P(\mathbf{x})=\sum_{i=1}^{n}\left\{\begin{array}{l}\text { if } x_{(i)}=x_{(i-1)} \text { and } \mathfrak{m}\left(A_{(i)}\right)=0 \\
x_{(i)}-x_{(i-1)}+\mathfrak{m}\left(A_{(i)}\right)-\left(x_{(i)}-x_{(i-1)}\right) \cdot \mathfrak{m}\left(A_{(i)}\right)\end{array}\right.$ otherwise \\
\hline
\end{tabular}

aggregation function which satisfies $M(0, y)=0$ for each $y \in[0,1]$.

Example 4.2: (i) Let $M:[0,1]^{2} \rightarrow[0,1]$ be any aggregation function. Then $S_{\mathfrak{m}}^{M}:[0,1]^{n} \rightarrow[0,1]$ is also an aggregation function, independently of $\mathfrak{m}$.

(ii) Consider the function $F, F(x, y)=x|2 y-1|$. Note that $F$ is a proper pre-aggregation function which satisfies the constraints of Proposition 4.2, and thus, for any $\mathfrak{m}$, the function $S_{\mathfrak{m}}^{F}:[0,1]^{n} \rightarrow[0,1], S_{\mathfrak{m}}^{F}(\mathbf{x})=\bigvee_{i=1}^{n} F\left(x_{(i)}, \mathfrak{m}\left(A_{(i)}\right)\right)$ is a preaggregation function (even an aggregation function thought $F$ is not).

For example, for $n=2, \mathfrak{m}(\{1\})=1 / 3, \mathfrak{m}(\{2\})=3 / 4$, we get

$$
S_{\mathfrak{m}}^{F}(x, y)= \begin{cases}x \vee \frac{y}{2} & \text { if } x \leq y, \\ y \vee \frac{x}{3} & \text { if } x>y\end{cases}
$$

\section{The Fuzzy Reasoning Method Using PRE-AGgREGATION FunCTIONS}

In this section, we present a generalization of the FRM proposed by Barrenechea et al. [24], using the proposed pre-aggregation functions, which are the result of combining different t-norms and fuzzy measures. To do so, we first explain the components of standard FRBCSs and then, the new FRM is introduced.

A classification problem consists of $m$ training examples $\mathbf{x}_{p}=\left(x_{p 1}, \ldots, x_{p n}, y_{p}\right)$, with $p=1, \ldots, m$, where $x_{p i}$, with $i=1, \ldots, n$, is the value of the $i$ th attribute variable and $y_{p} \in \mathbb{C}=\left\{C_{1}, C_{2}, \ldots, C_{M}\right\}$ is the label of the class of the $p$ th training example.

Among all the techniques used to face classification problems, one of the most used are the Fuzzy Rule-based Classification Systems (FRBCSs) [25], since they allow the inclusion of all the available information in the system modelling, generating an interpretable model and providing accurate results. The two main components of FRBCSs are:

(i) The Knowledge Base containing the Rule Base and the Data Base, where the fuzzy inference rules and the membership functions are stored, respectively. (ii) The Fuzzy Reasoning Mechanism, which is used to classify examples using the information available in the Knowledge Base.

The choice of the aggregation function plays a crucial role in FRBCSs [26], [27], since it determines the behaviour of the Fuzzy Reasoning Method (FRM) [28]. This is due to the fact that in the FRM the local information given by each fuzzy rule is aggregated to provide global information, which is associated with each class of the problem [28], [27], [29], [30], [31]. Finally, the example is assigned to the class having the maximum global information.

The usage of the maximum as the aggregation function in the FRM to obtain the global information is very common in the literature, which is known as the FRM of the winning rule [28], [27], [32], [33]. However, whenever one considers, for each class, just the information given by a single fuzzy rule having the highest compatibility with the example, the available information provided by the remaining fuzzy rules of the system is ignored.

Denote by $x_{p}=\left(x_{p 1}, \ldots, x_{p n}\right)$, the $n$-dimensional vector of attribute values corresponding to an example $\mathbf{x}_{p}$. The fuzzy rules that are used in this work are of the following form: Rule $R_{j}$ :

If $x_{p 1}$ is $A_{j 1}$ and $\ldots$ and $x_{p n}$ is $A_{j n}$ then $x_{p}$ in $C_{j}^{k}$ with $R W_{j}$,

where $R_{j}$ is the label of the $j$ th rule, $A_{j i}$ is an antecedent fuzzy set modelling a linguistic term, $C_{j}^{k}$ is the label of the consequent fuzzy set $C^{k}$ modelling the class associated to the rule $R_{j}$, with $k \in\{1, \ldots, M\}$, and $R W_{j} \in[0,1]$ is the rule weight [34].

Let $x_{p}=\left(x_{p 1}, \ldots, x_{p n}\right)$ be a new example to be classified, $L$ the number of rules in the rule base and $M$ the number of classes of the problem. The new FRM using pre-aggregation functions presents the following steps:

Matching degree: it is the strength of the activation of the if-part of the rules for the example $x_{p}$, which is computed 
using a t-norm $T^{\prime}:[0,1]^{n} \rightarrow[0,1]$ :

$\mu_{A_{j}}\left(x_{p}\right)=T^{\prime}\left(\mu_{A_{j 1}}\left(x_{p 1}\right), \ldots, \mu_{A_{j n}}\left(x_{p n}\right)\right)$, with $j=1, \ldots, L$.

Association degree: it is the association degree of the example $x_{p}$ with the class of each rule in the rule base, given by:

$b_{j}^{k}\left(x_{p}\right)=\mu_{A_{j}}\left(x_{p}\right) \cdot R W_{j}^{k}$, with $k=\operatorname{Class}\left(R_{j}\right), j=1, \ldots, L$.

Example classification soundness degree for all classes: in this step, we apply pre-aggregation functions (Equation (7)) to combine the association degrees calculated in the previous step, obtaining the classification soundness degrees, defined by:

$$
Y_{k}\left(x_{p}\right)=C_{\mathfrak{m}}^{T}\left(b_{1}^{k}\left(x_{p}\right), \ldots, b_{L}^{k}\left(x_{p}\right)\right), \text { with } k=1, \ldots, M,
$$

where $C_{\mathfrak{m}}^{T}$ is the obtained pre-aggregation, which is the result of combining a bivariate t-norm $T:[0,1]^{2} \rightarrow[0,1]$ and a fuzzy measure $\mathfrak{m}: 2^{N} \rightarrow[0,1]$.

Since, whenever $b_{i}^{k}\left(x_{p}\right)=0$, it holds that:

$$
\begin{gathered}
C_{\mathfrak{m}}^{T}\left(b_{1}^{k}\left(x_{p}\right), \ldots, b_{L}^{k}\left(x_{p}\right)\right) \\
=C_{\mathfrak{m}}^{T}\left(b_{1}^{k}\left(x_{p}\right), \ldots, b_{j-1}^{k}\left(x_{p}\right), b_{j+1}^{k}\left(x_{p}\right), \ldots, b_{L}^{k}\left(x_{p}\right)\right),
\end{gathered}
$$

then, for practical reasons, only $b_{j}^{k}>0$ are considered in Equation (12).

Classification: A decision function $F:[0,1]^{M} \rightarrow$ $\{1, \ldots, M\}$ defined over the example classification soundness degrees of all classes and determining the class corresponding to the maximum soundness degree is given by:

$$
F\left(Y_{1}, \ldots, Y_{M}\right)=\min _{k=1 \ldots M} k \text { such that } Y_{k}=\max _{w=1, \ldots, M}\left(Y_{w}\right) \text {. }
$$

In practical applications, it is sufficient to consider

$$
F\left(Y_{1}, \ldots, Y_{M}\right)=\underset{k=1, \ldots, M}{\arg \max }\left(Y_{k}\right) .
$$

Barrenechea et al. proposed to use the classical Choquet integral (product t-norm) instead of pre-aggregation in Equation (12). They also considered tuning the exponent of the power measure using an evolutionary algorithm [24]. Specifically they used the CHC evolutionary model [35], which was used to define the most suitable exponent to be used for each class. ${ }^{3}$ We denote this proposal as power measure genetically adjusted (Power_GA).

\section{ANALYSIS OF THE APPLICATION OF PRE-AGGREGATION FUNCTIONS IN CLASSIFICATION PROBLEMS}

This section is aimed at providing an application of preaggregation functions in real-world problems. Specifically, as introduced in Section V, we consider to introduce this new theory to extend the FRM proposed by Barrenechea et al. [24], which was applied to tackle classification problems.

The aim of the experimental study is to see whether the usage of pre-aggregation functions in this FRM allows the

\footnotetext{
${ }^{3}$ See [24] for a detailed explanation of the evolutionary algorithm.
}

results of the classical Choquet integral (product t-norm) to be enhanced. To do so, we test the performance of the FRM using 30 different pre-aggregation functions, which are all the possible combinations among the six t-norms shown in Table I and the five fuzzy measures (see Section II) considered in this paper. Finally, as it was done in [24], we also analyse if the best FRM (the best pre-aggregation) is able to enhance the results of the well-known FRM of the Winning Rule (WR), that is, the usage of the maximum to aggregate the information in the third step of the FRM described in Section V. Consequently, we want to show that the usage of pre-aggregation functions allows the results obtained with two classical averaging operators to be enhanced.

In the remainder of this section, we first explain the adopted experimental framework (Section VI-A) and then we present the results as well as their corresponding analysis (Section VI-B).

\section{A. Experimental framework}

We use 27 real world data-sets selected from the KEEL dataset repository [36]. Table III summarizes the properties of these datasets, showing, for each dataset, the identifier (Id.) as well as the name (Dataset), the number of instances (\#Inst), the number of attributes (\#Att) and the number of classes (\#Class). The magic, page-blocks, penbased, ring, satimage and twonorm datasets have been stratified sampled at $10 \%$ in order to reduce their size for training. Examples with missing values have been removed, e.g., in the wisconsin dataset.

TABLE III: Datasets used in this study

\begin{tabular}{llrrr}
\hline Id. & Dataset & \#Inst & \#Att & \#Class \\
\hline App & Appendiciticis & 106 & 7 & 2 \\
Bal & Balance & 625 & 4 & 3 \\
Ban & Banana & 5300 & 2 & 2 \\
Bnd & Bands & 365 & 19 & 2 \\
Bup & Bupa & 345 & 6 & 2 \\
Cle & Cleveland & 297 & 13 & 5 \\
Eco & Ecoli & 336 & 7 & 8 \\
Gla & Glass & 214 & 9 & 6 \\
Hab & Haberman & 306 & 3 & 2 \\
Hay & Hayes-Roth & 160 & 4 & 3 \\
Iri & Iris & 150 & 4 & 3 \\
Led & Led7digit & 500 & 7 & 10 \\
Mag & Magic & 1,902 & 10 & 2 \\
New & Newthyroid & 215 & 5 & 3 \\
Pag & Pageblocks & 5,472 & 10 & 5 \\
Pho & Phoneme & 5,404 & 5 & 2 \\
Pim & Pima & 768 & 8 & 2 \\
Rin & Ring & 740 & 20 & 2 \\
Sah & Saheart & 462 & 9 & 2 \\
Sat & Satimage & 6,435 & 36 & 7 \\
Seg & Segment & 2,310 & 19 & 7 \\
Tit & Titanic & 2,201 & 3 & 2 \\
Two & Twonorm & 740 & 20 & 2 \\
Veh & Vehicle & 846 & 18 & 4 \\
Win & Wine & 178 & 13 & 3 \\
Wis & Wisconsin & 683 & 11 & 2 \\
Yea & Yeast & 1,484 & 8 & 10 \\
\hline & & & &
\end{tabular}

We adopt the model proposed in [24], [37], [38], that is, a 5-fold cross-validation model, where a dataset is split in five partitions randomly, each partition with $20 \%$ of the examples, and a combination of four of them is then used for training and the other is used for testing. This process is repeated five 
times by using a different partition to test the system each time. For each partition the output is computed as the mean of the numbers of correctly classified examples divided by the total number of examples for each partition, that is, the accuracy rate. Then, we consider the average result of the five partitions as the final classification rate of the algorithm. This procedure is a standard for testing the performance of classifiers [39], [40].

We use FARC-HD [10], which is short for Fuzzy Association Rule-based Classification model for High Dimensional problems, to accomplish the fuzzy rule learning process. We have considered the following configuration: the product $\mathrm{t}-$ norm as the conjunction operator $T^{\prime}$, the Certainty Factor as the rule weight $R W_{j}, 5$ linguistic labels per variable, 0.05 for the minimum support, 0.8 as the threshold for the confidence, the depth of the search trees is limited to 3 and the parameter determining the number of fuzzy rules that cover each example, $k_{t}$, is set to 2 . For the genetic process, we have used populations composed of 50 individuals, 30 bits per gen for the Gray codification (for incest prevention) and 20,000 as the maximum number of iterations. Finally, for the Dirac fuzzy measure, the value of the variable $i$ used to decide if $i \in A$, for $A \subseteq N=\{0, \ldots, n\}$, we adopt the median value, given by,

$$
i= \begin{cases}\frac{n+1}{2} & \text { if } n \text { is odd } \\ \frac{n}{2}+1 & \text { if } n \text { is even. }\end{cases}
$$

In order to give statistical support to the analysis of the results we consider the usage of hypothesis validation techniques [41], [42]. Specifically, we use non-parametric tests, since the initial conditions that guarantee the reliability of the parametric tests cannot be performed [43].

In fact, we use the aligned Friedman test [44] to detect statistical differences among a group of results and to show how good a method is with respect to the others. In this method, the algorithm achieving the lowest average ranking is the best one. Furthermore, we apply the post-hoc Holm's test [45] to study whether the best method rejects the equality hypothesis with respect to its partners. The post-hoc procedure allows us to know whether a hypothesis of comparison could be rejected at a specified level of significance $\alpha$. Specifically, we compute the adjusted $p$-value (APV) to take into account that multiple tests are conducted. As a result, we can directly compare the APV with the level of significance $\alpha$ so as to be able to reject the null hypothesis.

Finally, we also consider the usage of the Wilcoxon test [46] in order to perform pair-wise comparisons.

\section{B. Experimental Results}

The summary of the results provided by all the different configurations of the FRM, i.e. all the pre-aggregation functions, are introduced in Table IV. Each column of this table shows the results obtained using the fuzzy measure reported in its top cell using the six t-norms, which are shown by rows. The number in each cell is the average of the accuracy rate obtained in the 27 datasets by the corresponding preaggregation function. The best result for each fuzzy measure is highlighted in bold-face. The number in brackets is the number of datasets in which each t-norm has obtained the best performance for each fuzzy measure (ties are excluded). The detailed results obtained in each dataset are available in A.

TABLE IV: Averaged results obtained by the different preaggregation functions considered in the study.

\begin{tabular}{cccccc}
\hline & Uniform & Dirac & Wmean & OWA & Power_GA \\
\hline Product & $78.68(7)$ & $\mathbf{7 8 . 0 1 ( 3 )}$ & $78.12(4)$ & $77.33(4)$ & $78.55(5)$ \\
Minimum & $78.85(7)$ & $77.81(0)$ & $78.75(7)$ & $78.33(10)$ & $79.00(7)$ \\
Eukasiewicz & $76.61(1)$ & $77.81(1)$ & $76.92(0)$ & $76.44(1)$ & $78.14(0)$ \\
Drastic & $76.66(0)$ & $77.81(0)$ & $76.66(1)$ & $76.66(2)$ & $76.66(1)$ \\
Nilpotent & $76.88(1)$ & $77.81(0)$ & $76.76(3)$ & $76.60(1)$ & $78.78(5)$ \\
Hamacher & $\mathbf{7 9 . 1 6 ( 8 )}$ & $77.81(1)$ & $\mathbf{7 9 . 1 9 ( 1 0 )}$ & $\mathbf{7 8 . 6 1 ( 7 )}$ & $\mathbf{7 9 . 4 2 ( 7 )}$ \\
\hline
\end{tabular}

From these results we can observe two situations:

- The performance of the product, minimum and Hamacher is in general clearly better than that of Łukasiewicz, Drastic product and Nilpotent minimum.

- The performance of all the t-norms using the Dirac's measure is almost the same.

The reason implying the low performance of Łukasiewicz, Drastic product and Nilpotent product is that after aggregating a set of values, the obtained one is similar to that obtained if we aggregated them using the minimum function (not the pre-aggregation associated with the minimum), which usually obtains poor results. The explanation is as follows: let $x$ and $y$ be the result of the fuzzy measure and the subtraction of the elements to be aggregated using the Choquet integral, respectively.

- Łukasiewicz: $x+y-1$ is lower than 0 on half of its domain. Therefore, most of the time we do not add anything, which implies obtaining the minimum or a value close to it.

- Drastic product: the value of the fuzzy measure is never 1 (except when we have all the elements) and it is very difficult to have a difference between two values to be aggregated equal to 1 . Therefore, most of the time we add 0 .

- Nilpotent minimum: in the same way than Łukasiewicz, on half of the domain $x+y$ is greater than 1 . Consequently, we also add 0 most of the times.

Regarding the behaviour of the Dirac's measure, the similar behaviour among all the t-norms is due to the fact that this measure returns always either 1 or 0 . Furthermore, it is known that $T\left(x_{(i)}-x_{(i-1)}, 0\right)=0$ and $T\left(x_{(i)}-x_{(i-1)}, 1\right)=x_{(i)}-$ $x_{(i-1)}$, for any t-norm $T$. Consequently, the selected t-norm $T$ does not have a great influence on the results of the preaggregation functions.

Due to the aforementioned poor results obtained when applying Łukasiewicz, Drastic product and Nilpotent minimum, we focus the remainder of the analysis on the product, minimum and Hamacher t-norms.

From the results on Table IV, we can observe that, with the exception of the Dirac's fuzzy measure, the results of the Hamacher t-norm are better than those of the minimum t-norm, which in turn are better than the ones of the product. This trend is also present, in general, on the number of datasets in which each of these t-norms obtain the best result. 
In order to support the previous findings, we carry out a statistical test to compare, for each fuzzy measure, the product, minimum and Hamacher t-norms. To do so, we have used the Aligned Friedman test as well as the Holm's post-hoc test. The results of these statistical techniques are reported in Table V, where in each column we find the different fuzzy measures whereas the three t-norms are shown in rows. The number in each cell is the average rank computed with the aligned Friedman test and the number in brackets is the APV computed with the Holm's test. The best t-norm for each fuzzy measure is the one with the less rank, which stressed in bold-face, whereas the APV is underlined in case of statistical differences in favour to the best t-norm.

TABLE V: Aligned Friedman and Holm tests to compare the different pre-aggregation functions.

\begin{tabular}{|c|c|c|c|c|c|}
\hline & Uniform & Dirac & WMean & OWA & Power_GA \\
\hline Product & $42.94(0.21)$ & 38.13 & $51.09(\underline{0.002})$ & $53.91(\underline{0.003})$ & $50.78(\underline{0.004})$ \\
\hline Minimum & $45.13(0.21)$ & $43.38(0.771)$ & $42.13(\overline{0.054})$ & $35.24(\overline{0.828})$ & $41.20(\overline{0.112})$ \\
\hline Hamacher & 50.22 & $41.18(0.771)$ & 29.78 & 33.85 & 31.02 \\
\hline
\end{tabular}

From the results in Table $\mathrm{V}$, we can observe that the usage of the Hamacher t-norm provides the best behaviour for all the fuzzy measures, with the exception of the one defined by Dirac due to the previous mentioned behaviour. In fact, we find statistical differences with respect to the product when using the additive (WMean), symmetric (OWA) and Power_GA fuzzy measures and a low APV when using the uniform measure. Therefore, we can conclude that the usage of the Hamacher t-norm allows us to enhance the results of the product.

Furthermore, we also want to analyse if the minimum is also appropriate when compared with the usage of the product. To do so, we compare, for each fuzzy measure, the results provided by the product versus the ones of the minimum. To perform these comparisons, we have applied the Wilcoxon's test to conduct such pair-wise comparisons. The obtained results are introduced in Table VI, where we can observe that when using the additive (WMean), symmetric (OWA) and Power_GA fuzzy measures there is a trend in favour to the minimum whereas in the two remainder fuzzy measures the behaviour of these two t-norms is similar.

TABLE VI: Wilcoxon Test to compare the product $\left(R^{+}\right)$ versus the minimum $\left(R^{-}\right)$.

\begin{tabular}{lccc}
\hline Comparison & $R^{+}$ & $R^{-}$ & p-value \\
\hline Uniform+Prod vs. Uniform+Min & 195.5 & 182.5 & 0.925 \\
Dirac+Prod vs. Dirac+Min & 214 & 164 & 0.625 \\
WMean+Prod vs. WMean+Min & 135.5 & 242.5 & 0.200 \\
OWA+Prod vs. OWA+Min & 107.5 & 270.5 & 0.004 \\
Power_GA+Prod vs. Power_GA+Min & 132 & 249 & 0.148 \\
\hline
\end{tabular}

Finally, we want to study whether the results obtained by the best pre-aggregation function are able to improve those provided by the well-known FRM of the WR, that is, the usage of the maximum to aggregate the information. According to Table IV, we select the pre-aggregation function resulting of the combination among the Power_GA fuzzy measure and the Hamacher t-norm (Power_GA+Ham), since it provides the best average result. The results provided by this preaggregation function as well as those obtained with the WR are reported in Table VII, where the best result for each dataset is highlighted in bold-face. From these results, it can be observed that the global behaviour of Power_GA+Ham is better than that of the WR. This is due to the fact that Power_GA+Ham provides the best result in 17 out of the 27 datasets considered in the study. We also apply the Wilcoxon's test to support these findings, whose obtained results are shown in Table VIII. According to the statistical results, we can confirm with a high level of confidence that the usage of Power_GA+Ham is better than that of the WR.

TABLE VII: Results in testing provided by Card_GA+Ham and WR.

\begin{tabular}{ccc}
\hline Dataset & WR & Power_GA+Ham \\
\hline App & $\mathbf{8 4 . 8 9}$ & 82.99 \\
Bal & 82.08 & $\mathbf{8 2 . 7 2}$ \\
Ban & 84.30 & $\mathbf{8 5 . 9 6}$ \\
Bnd & 68.56 & $\mathbf{7 2 . 1 3}$ \\
Bup & 61.16 & $\mathbf{6 5 . 8 0}$ \\
Cle & 55.23 & $\mathbf{5 5 . 5 8}$ \\
Eco & 75.61 & $\mathbf{8 0 . 0 7}$ \\
Gal & $\mathbf{6 3 . 1 1}$ & 63.10 \\
Hab & 71.22 & $\mathbf{7 2 . 2 1}$ \\
Hay & 79.46 & $\mathbf{7 9 . 4 9}$ \\
Iri & $\mathbf{9 4 . 6 7}$ & 93.33 \\
Led & $\mathbf{6 9 . 8 0}$ & 68.60 \\
Mag & 79.60 & $\mathbf{7 9 . 7 6}$ \\
New & 94.42 & $\mathbf{9 5 . 3 5}$ \\
Pag & $\mathbf{9 4 . 5 2}$ & 94.34 \\
Pho & 82.01 & $\mathbf{8 3 . 8 3}$ \\
Pim & $\mathbf{7 5 . 3 8}$ & 73.44 \\
Rin & $\mathbf{9 0 . 0 0}$ & 88.79 \\
Sah & 67.31 & $\mathbf{7 0 . 7 7}$ \\
Sat & $\mathbf{8 0 . 4 0}$ & $\mathbf{8 0 . 4 0}$ \\
Seg & 92.99 & $\mathbf{9 3 . 3 3}$ \\
Tit & $\mathbf{7 8 . 8 7}$ & $\mathbf{7 8 . 8 7}$ \\
Two & 84.32 & $\mathbf{8 5 . 2 7}$ \\
Veh & 67.62 & $\mathbf{6 8 . 2 0}$ \\
Win & 94.36 & $\mathbf{9 6 . 6 3}$ \\
Wis & 96.49 & $\mathbf{9 6 . 7 8}$ \\
Yea & $\mathbf{5 6 . 5 4}$ & 56.53 \\
\hline Mean & 78.70 & $\mathbf{7 9 . 4 2}$ \\
\hline
\end{tabular}

TABLE VIII: Wilcoxon Test to compare the power measure genetically adjusted method with the Hamacher t-norm $\left(R^{+}\right)$ versus the classical FRM of the Winning Rule $\left(R^{-}\right)$.

\begin{tabular}{lccc}
\hline Comparison & $R^{+}$ & $R^{-}$ & p-value \\
\hline Power_GA+Ham vs. WR & 267.5 & 110.5 & 0.06 \\
\hline
\end{tabular}

\section{CONCLUSION}

In this paper, based on the notion of an aggregation function, we have introduced the concept of a pre-aggregation function. We have described three construction methods for such functions. In particular, one of them derives from the Choquet integral by using other t-norms in the place of the product t-norm considered in the standard definition of the Choquet integral. Furthermore, we have proposed to apply this specific instance of pre-aggregation in the FRM of FRBCSs 
to aggregate the local information given by each fuzzy rule of the system.

In the experimental study we have shown that the usage of the Hamacher or even the minimum t-norms allows one to improve the results obtained when applying the classical Choquet integral, that is, when using the product t-norm. Moreover, we have checked that the pre-aggregation providing the best results, which is obtained combining the Hamacher t-norm and the power measure genetically learnt, enhances the results achieved by the well-known FRM of the winning rule, that is, applying the maximum as the aggregation function. Therefore, the pre-aggregation functions introduced in this paper can offer greater flexibility for FRBCSs, enlarging the scope of the application of the approach proposed by Barrenechea et al. [24].

Future work is concerned with the study of the properties satisfied by the pre-aggregation functions, and the usage of overlap functions [6], [7], [47], [48], [49] for the generalization of the Choquet integral, also using a fuzzy interval approach [50], [51], [52], [53], [54], as, e.g., in [55], [33], [31].

\section{APPENDIX}

The tables in this Appendix present the obtained results in each dataset considering the different t-norms, for each fuzzy measure. Each table contains the results obtained with a different fuzzy measure:

- Table IX: results of the uniform measure for the six tnorms.

- Table X: results of the Dirac's fuzzy measure for the six t-norms.

- Table XI: results of an additive fuzzy measure for the six t-norms.

- Table XII: results of the ordered weighted averaging fuzzy measure for the six t-norms.

- Table XIII: results of the genetic uniform fuzzy measure for the six t-norms.

The structure of these 5 tables is as follows: in each row we find a dataset and in each column we introduce a different $\mathrm{t}$ norm. The best result for each dataset is stressed in boldface.

\section{REFERENCES}

[1] G. Beliakov, A. Pradera, and T. Calvo, Aggregation Functions: A Guide for Practitioners. Berlin: Springer, 2007.

[2] M. Grabisch, J. Marichal, R. Mesiar, and E. Pap, Aggregation Functions. Cambridge: Cambridge University Press, 2009.

[3] H. Bustince, E. Barrenechea, T. Calvo, S. James, and G. Beliakov, "Consensus in multi-expert decision making problems using penalty functions defined over a cartesian product of lattices," Information Fusion, vol. 17, pp. 56-64, 2014.

[4] H. Bustince, M. Pagola, R. Mesiar, E. Hüllermeier, and F. Herrera, "Grouping, overlaps, and generalized bientropic functions for fuzzy modeling of pairwise comparisons," IEEE Transactions on Fuzzy Systems, vol. 20, no. 3, pp. 405-415, 2012

[5] H. Bustince, J. Montero, and R. Mesiar, "Migrativity of aggregation operators," Fuzzy Sets and Systems, vol. 160, no. 6, pp. 766-777, 2009.

[6] H. Bustince, J. Fernandez, R. Mesiar, J. Montero, and R. Orduna, "Overlap functions," Nonlinear Analysis, vol. 72, no. 3-4, pp. 14881499, 2010.

[7] A. Jurio, H. Bustince, M. Pagola, A. Pradera, and R. Yager, "Some properties of overlap and grouping functions and their application to image thresholding," Fuzzy Sets and Systems, vol. 229, pp. 69 - 90, 2013.
TABLE IX: Detailed results in testing using the uniform measure.

\begin{tabular}{ccccccc}
\hline Dataset & Product & Minimum & Lukasiewicz & Drastic & Nilpotent & Hamacher \\
\hline App & 86.80 & 84.89 & $\mathbf{8 7 . 7 5}$ & 83.03 & 82.12 & 85.89 \\
Bal & 78.24 & $\mathbf{8 2 . 2 4}$ & 75.04 & 76.80 & 77.12 & 80.96 \\
Ban & $\mathbf{8 4 . 4 5}$ & 83.38 & 82.70 & 82.72 & 81.91 & 84.19 \\
Bnd & 64.00 & $\mathbf{7 0 . 2 4}$ & 64.07 & 65.56 & 63.81 & 69.96 \\
Bup & 64.35 & 63.19 & 63.77 & 63.48 & 65.22 & $\mathbf{6 5 . 8 0}$ \\
Cle & $\mathbf{5 7 . 5 7}$ & 55.55 & 55.24 & 56.89 & 52.51 & 56.90 \\
Eco & 78.28 & 76.20 & 72.91 & 75.61 & 75.61 & $\mathbf{7 9 . 1 7}$ \\
Gal & $\mathbf{6 5 . 9 0}$ & 63.58 & 62.62 & 62.17 & 62.16 & 64.47 \\
Hab & $\mathbf{7 4 . 5 0}$ & 72.53 & 73.51 & 73.20 & 73.84 & 72.87 \\
Hay & $\mathbf{8 1 . 0 0}$ & 78.69 & 78.77 & 78.77 & 79.52 & 79.49 \\
Iri & 94.00 & 94.00 & 94.00 & 92.67 & $\mathbf{9 4 . 6 7}$ & 93.33 \\
Led & 68.20 & 68.80 & 67.40 & 67.00 & 68.40 & $\mathbf{6 9 . 0 0}$ \\
Mag & 79.02 & 79.49 & 76.50 & 77.28 & 76.97 & $\mathbf{8 0 . 6 5}$ \\
New & 94.42 & $\mathbf{9 5 . 3 5}$ & 93.02 & 92.56 & 92.56 & 94.88 \\
Pag & 94.16 & 93.80 & 93.61 & $\mathbf{9 4 . 3 4}$ & 94.16 & 94.34 \\
Pho & 83.14 & 81.92 & 80.18 & 79.70 & 79.81 & $\mathbf{8 3 . 3 3}$ \\
Pim & 72.26 & $\mathbf{7 4 . 7 4}$ & 71.62 & 72.65 & 72.40 & 74.48 \\
Rin & 85.81 & $\mathbf{8 8 . 2 4}$ & 78.38 & 78.11 & 79.59 & 87.43 \\
Sah & $\mathbf{7 0 . 9 7}$ & 70.55 & 68.83 & 68.61 & 69.70 & 69.68 \\
Sat & $\mathbf{8 4 . 5 0}$ & 81.80 & 77.76 & 78.38 & 76.36 & 79.47 \\
Seg & 92.60 & 93.07 & 90.00 & 90.69 & 89.74 & $\mathbf{9 3 . 2 5}$ \\
Tit & $\mathbf{7 8 . 8 7}$ & $\mathbf{7 8 . 8 7}$ & $\mathbf{7 8 . 8 7}$ & $\mathbf{7 8 . 8 7}$ & $\mathbf{7 8 . 8 7}$ & $\mathbf{7 8 . 8 7}$ \\
Two & 80.54 & $\mathbf{8 3 . 2 4}$ & 77.84 & 77.70 & 76.22 & 82.70 \\
Veh & 63.53 & 68.56 & 66.78 & 64.89 & 65.72 & $\mathbf{6 9 . 0 3}$ \\
Win & 94.37 & 93.81 & 88.71 & 88.73 & 95.49 & $\mathbf{9 5 . 5 1}$ \\
Wis & 95.90 & $\mathbf{9 6 . 0 5}$ & 95.02 & 95.32 & 94.44 & 95.76 \\
Yea & 56.94 & 56.26 & 53.44 & 53.97 & $\mathbf{5 6 . 9 4}$ & 56.00 \\
\hline Mean & 78.68 & 78.85 & 76.61 & 76.66 & 76.88 & $\mathbf{7 9 . 1 6}$ \\
\hline & & & & & & \\
\hline & & & &
\end{tabular}

TABLE X: Detailed results in testing using the Dirac's measure.

\begin{tabular}{ccccccc}
\hline Dataset & Product & Minimum & Lukasiewicz & Drastic & Nilpotent & Hamacher \\
\hline Dataset & Product & Minimum & Lukasewitz & Drastic & Nilpo & Hamacher \\
App & $\mathbf{8 0 . 1 7}$ & $\mathbf{8 0 . 1 7}$ & $\mathbf{8 0 . 1 7}$ & $\mathbf{8 0 . 1 7}$ & $\mathbf{8 0 . 1 7}$ & $\mathbf{8 0 . 1 7}$ \\
Bal & $\mathbf{7 8 . 2 4}$ & $\mathbf{7 8 . 2 4}$ & 77.60 & $\mathbf{7 8 . 2 4}$ & 78.08 & $\mathbf{7 8 . 2 4}$ \\
Ban & $\mathbf{8 4 . 0 9}$ & $\mathbf{8 4 . 0 9}$ & $\mathbf{8 4 . 0 9}$ & $\mathbf{8 4 . 0 9}$ & $\mathbf{8 4 . 0 9}$ & $\mathbf{8 4 . 0 9}$ \\
Bnd & $\mathbf{7 0 . 6 7}$ & 65.97 & 65.97 & 65.97 & 65.97 & 65.97 \\
Bup & $\mathbf{6 4 . 0 6}$ & $\mathbf{6 4 . 0 6}$ & $\mathbf{6 4 . 0 6}$ & $\mathbf{6 4 . 0 6}$ & $\mathbf{6 4 . 0 6}$ & $\mathbf{6 4 . 0 6}$ \\
Cle & $\mathbf{5 5 . 5 6}$ & $\mathbf{5 5 . 5 6}$ & $\mathbf{5 5 . 5 6}$ & $\mathbf{5 5 . 5 6}$ & $\mathbf{5 5 . 5 6}$ & $\mathbf{5 5 . 5 6}$ \\
Eco & $\mathbf{7 7 . 7 0}$ & $\mathbf{7 7 . 7 0}$ & $\mathbf{7 7 . 7 0}$ & $\mathbf{7 7 . 7 0}$ & $\mathbf{7 7 . 7 0}$ & $\mathbf{7 7 . 7 0}$ \\
Gal & $\mathbf{6 4 . 9 8}$ & $\mathbf{6 4 . 9 8}$ & $\mathbf{6 4 . 9 8}$ & $\mathbf{6 4 . 9 8}$ & $\mathbf{6 4 . 9 8}$ & $\mathbf{6 4 . 9 8}$ \\
Hab & $\mathbf{7 1 . 2 3}$ & $\mathbf{7 1 . 2 3}$ & $\mathbf{7 1 . 2 3}$ & $\mathbf{7 1 . 2 3}$ & $\mathbf{7 1 . 2 3}$ & $\mathbf{7 1 . 2 3}$ \\
Hay & 78.69 & 78.69 & $\mathbf{7 9 . 4 6}$ & 78.69 & 78.69 & 78.69 \\
Iri & $\mathbf{9 3 . 3 3}$ & $\mathbf{9 3 . 3 3}$ & $\mathbf{9 3 . 3 3}$ & $\mathbf{9 3 . 3 3}$ & $\mathbf{9 3 . 3 3}$ & $\mathbf{9 3 . 3 3}$ \\
Led & 68.00 & 68.00 & 68.00 & 68.00 & 68.00 & $\mathbf{6 8 . 2 0}$ \\
Mag & $\mathbf{7 7 . 8 6}$ & $\mathbf{7 7 . 8 6}$ & $\mathbf{7 7 . 8 6}$ & $\mathbf{7 7 . 8 6}$ & $\mathbf{7 7 . 8 6}$ & 77.86 \\
New & $\mathbf{9 3 . 0 2}$ & $\mathbf{9 3 . 0 2}$ & $\mathbf{9 3 . 0 2}$ & $\mathbf{9 3 . 0 2}$ & $\mathbf{9 3 . 0 2}$ & $\mathbf{9 3 . 0 2}$ \\
Pag & $\mathbf{9 4 . 5 2}$ & $\mathbf{9 4 . 5 2}$ & $\mathbf{9 4 . 5 2}$ & $\mathbf{9 4 . 5 2}$ & $\mathbf{9 4 . 5 2}$ & $\mathbf{9 4 . 5 2}$ \\
Pho & $\mathbf{8 2 . 3 3}$ & $\mathbf{8 2 . 3 3}$ & $\mathbf{8 2 . 3 3}$ & $\mathbf{8 2 . 3 3}$ & $\mathbf{8 2 . 3 3}$ & $\mathbf{8 2 . 3 3}$ \\
Pim & $\mathbf{7 2 . 5 2}$ & $\mathbf{7 2 . 5 2}$ & $\mathbf{7 2 . 5 2}$ & $\mathbf{7 2 . 5 2}$ & $\mathbf{7 2 . 5 2}$ & $\mathbf{7 2 . 5 2}$ \\
Rin & $\mathbf{8 4 . 5 9}$ & $\mathbf{8 4 . 5 9}$ & $\mathbf{8 4 . 5 9}$ & $\mathbf{8 4 . 5 9}$ & $\mathbf{8 4 . 5 9}$ & $\mathbf{8 4 . 5 9}$ \\
Sah & $\mathbf{7 0 . 9 7}$ & 68.82 & 68.82 & 68.82 & 68.82 & 68.82 \\
Sat & $\mathbf{7 9 . 8 4}$ & 78.85 & 78.85 & 78.85 & 78.85 & 78.85 \\
Seg & $\mathbf{9 1 . 0 4}$ & $\mathbf{9 1 . 0 4}$ & $\mathbf{9 1 . 0 4}$ & $\mathbf{9 1 . 0 4}$ & $\mathbf{9 1 . 0 4}$ & $\mathbf{9 1 . 0 4}$ \\
Tit & $\mathbf{7 9 . 0 6}$ & $\mathbf{7 9 . 0 6}$ & $\mathbf{7 9 . 0 6}$ & $\mathbf{7 9 . 0 6}$ & $\mathbf{7 9 . 0 6}$ & $\mathbf{7 9 . 0 6}$ \\
Two & $\mathbf{8 2 . 3 0}$ & $\mathbf{8 2 . 3 0}$ & $\mathbf{8 2 . 3 0}$ & $\mathbf{8 2 . 3 0}$ & $\mathbf{8 2 . 3 0}$ & $\mathbf{8 2 . 3 0}$ \\
Veh & 62.35 & $\mathbf{6 4 . 6 6}$ & $\mathbf{6 4 . 6 6}$ & $\mathbf{6 4 . 6 6}$ & $\mathbf{6 4 . 6 6}$ & $\mathbf{6 4 . 6 6}$ \\
Win & $\mathbf{9 6 . 0 6}$ & $\mathbf{9 6 . 0 6}$ & $\mathbf{9 6 . 0 6}$ & $\mathbf{9 6 . 0 6}$ & $\mathbf{9 6 . 0 6}$ & $\mathbf{9 6 . 0 6}$ \\
Wis & $\mathbf{9 5 5 . 9 0}$ & $\mathbf{9 5 . 9 0}$ & $\mathbf{9 5 . 9 0}$ & $\mathbf{9 5 . 9 0}$ & $\mathbf{9 5 . 9 0}$ & $\mathbf{9 5 . 9 0}$ \\
Yea & $\mathbf{5 7 . 2 1}$ & $\mathbf{5 7 . 2 1}$ & $\mathbf{5 7 . 2 1}$ & $\mathbf{5 7 . 2 1}$ & $\mathbf{5 7 . 2 1}$ & $\mathbf{5 7 . 2 1}$ \\
\hline Mean & $\mathbf{7 8 . 0 1}$ & 77.81 & 77.81 & 77.81 & 77.80 & 77.81 \\
\hline
\end{tabular}

[8] T. Wilkin and G. Beliakov, "Weakly monotone aggregation functions," International Journal of Intelligent Systems, vol. 30, pp. 144-169, 2015.

[9] H. Bustince, A. Kolesárová, J. Fernandez, and R. Mesiar, "Directional monotonicity of fusion functions," European Journal of Operational Research, in press, doi.10.1016/j.ejor.2015.01.018.

[10] J. Alcala-Fdez, R. Alcala, and F. Herrera, "A fuzzy association rulebased classification model for high-dimensional problems with genetic rule selection and lateral tuning," IEEE Transactions on Fuzzy Systems, vol. 19 , no. 5, pp. 857-872, 2011.

[11] G. Mayor and E. Trillas, "On the representation of some aggregation functions," in Proceedings of IEEE International Symposium on Multiple-Valued Logic. Los Alamitos: IEEE, 1986, pp. 111-114.

[12] E. P. Klement, R. Mesiar, and E. Pap, Triangular Norms. Dordrecht: Kluwer Academic Publisher, 2000.

[13] M. Grabisch and C. Labreuche, "A decade of application of the Choquet and Sugeno integrals in multi-criteria decision aid," Annals of Operations Research, vol. 175, no. 1, pp. 247-286, 2010.

[14] J.-L. Marichal, "Aggregation of interacting criteria by means of the discrete Choquet integral," in Aggregation Operators, ser. Studies in Fuzziness and Soft Computing, T. Calvo, G. Mayor, and R. Mesiar, Eds. Physica-Verlag HD, 2002, vol. 97, pp. 224-244.

[15] R. R. Yager, "On ordered weighted averaging aggregation operators in multicriteria decision making," Systems, Man and Cybernetics, IEEE 
TABLE XI: Detailed results in testing using an additive measure (WMean).

\begin{tabular}{ccccccc}
\hline Dataset & Product & Minimum & Lukasiewicz & Drastic & Nilpotent & Hamacher \\
\hline Dataset & Product & Minimum & Lukasewitz & Drastic & Nilpo & Hamacher \\
App & 82.08 & 83.94 & 82.08 & 83.03 & 83.98 & $\mathbf{8 5 . 8 4}$ \\
Bal & 78.08 & $\mathbf{8 1 . 6 0}$ & 75.52 & 76.80 & 74.56 & 81.12 \\
Ban & 83.85 & 84.02 & 83.30 & 82.72 & 82.11 & $\mathbf{8 4 . 4 7}$ \\
Bnd & 61.33 & $\mathbf{7 1 . 3 2}$ & 69.83 & 65.56 & 68.20 & 67.99 \\
Bup & 64.35 & 61.16 & 65.22 & 63.48 & $\mathbf{6 5 . 8 0}$ & 65.51 \\
Cle & 57.56 & 55.24 & 54.86 & 56.89 & 56.22 & $\mathbf{5 7 . 9 2}$ \\
Eco & $\mathbf{7 9 . 4 6}$ & 78.86 & 73.53 & 75.61 & 76.19 & 76.49 \\
Gal & 63.54 & $\mathbf{6 4 . 0 5}$ & 62.62 & 62.17 & 63.57 & 64.02 \\
Hab & 72.54 & 70.91 & 73.19 & $\mathbf{7 3 . 2 0}$ & 70.24 & 72.21 \\
Hay & 77.98 & 78.69 & 78.77 & 78.77 & $\mathbf{7 9 . 5 2}$ & 79.49 \\
Iri & 93.33 & 94.00 & 93.33 & 92.67 & $\mathbf{9 4 . 0 0}$ & 93.33 \\
Led & 68.40 & 68.20 & 67.80 & 67.00 & 67.80 & $\mathbf{6 9 . 4 0}$ \\
Mag & 80.55 & $\mathbf{8 0 . 7 6}$ & 76.08 & 77.28 & 76.97 & 80.02 \\
New & 93.95 & $\mathbf{9 4 . 8 8}$ & 92.56 & 92.56 & 92.56 & 94.42 \\
Pag & 94.44 & 94.16 & 93.97 & 94.34 & $\mathbf{9 4 . 7 1}$ & 94.34 \\
Pho & $\mathbf{8 2 . 5 1}$ & 82.11 & 79.44 & 79.70 & 79.90 & 82.25 \\
Pim & 73.56 & 74.86 & 72.40 & 72.65 & 71.75 & $\mathbf{7 5 . 7 8}$ \\
Rin & 85.68 & 88.24 & 76.89 & 78.11 & 78.65 & $\mathbf{8 8 . 7 8}$ \\
Sah & 65.59 & 69.27 & 70.57 & 68.61 & 67.97 & $\mathbf{7 1 . 2 1}$ \\
Sat & $\mathbf{8 1 . 4 0}$ & 78.23 & 78.85 & 78.38 & 77.91 & 79.78 \\
Seg & 92.12 & 92.21 & 90.00 & 90.69 & 89.61 & $\mathbf{9 2 . 8 6}$ \\
Tit & $\mathbf{7 8 . 8 7}$ & $\mathbf{7 8 . 8 7}$ & $\mathbf{7 8 . 8 7}$ & $\mathbf{7 8 . 8 7}$ & $\mathbf{7 8 . 8 7}$ & $\mathbf{7 8 . 8 7}$ \\
Two & 82.03 & 83.65 & 77.97 & 77.70 & 75.14 & $\mathbf{8 5 . 4 1}$ \\
Veh & $\mathbf{7 0 . 0 0}$ & 68.67 & 64.89 & 64.89 & 64.42 & 69.86 \\
Win & 94.40 & $\mathbf{9 5 . 4 8}$ & 94.37 & 88.73 & 92.11 & 93.81 \\
Wis & 95.76 & 95.75 & 95.46 & 95.32 & 95.02 & $\mathbf{9 7 . 0 7}$ \\
Yea & 56.00 & $\mathbf{5 7 . 2 1}$ & 54.58 & 53.97 & 54.79 & 56.00 \\
\hline Mean & 78.12 & 78.75 & 76.92 & 76.66 & 76.76 & $\mathbf{7 9 . 1 9}$ \\
\hline
\end{tabular}

TABLE XII: Detailed results in testing using a symmetric measure (OWA).

\begin{tabular}{ccccccc}
\hline Dataset & Product & Minimum & Lukasiewicz & Drastic & Nilpotent & Hamacher \\
\hline Dataset & Product & Minimum & Lukasewitz & Drastic & Nilpo & Hamacher \\
App & 83.03 & 82.99 & 82.12 & 83.03 & $\mathbf{8 8 . 6 6}$ & 84.85 \\
Bal & 78.88 & $\mathbf{8 2 . 5 6}$ & 77.28 & 76.80 & 74.72 & 80.80 \\
Ban & $\mathbf{8 4 . 5 5}$ & 83.23 & 82.21 & 82.72 & 82.79 & 83.23 \\
Bnd & 61.33 & 68.26 & 64.95 & 65.56 & 66.61 & $\mathbf{6 8 . 5 6}$ \\
Bup & 62.90 & 61.74 & 65.51 & 63.48 & 63.19 & $\mathbf{6 6 . 6 7}$ \\
Cle & 53.20 & 55.23 & 53.54 & $\mathbf{5 6 . 8 9}$ & 55.56 & 56.21 \\
Eco & $\mathbf{7 6 . 2 0}$ & 75.90 & 73.82 & 75.61 & 75.03 & 74.12 \\
Gal & 63.09 & 62.64 & 61.23 & 62.17 & 64.03 & $\mathbf{6 7 . 7 4}$ \\
Hab & 73.19 & 71.89 & $\mathbf{7 4 . 4 8}$ & 73.20 & 72.88 & 71.57 \\
Hay & 78.75 & $\mathbf{7 9 . 4 9}$ & 78.77 & 78.77 & 78.77 & 79.49 \\
Iri & 92.00 & $\mathbf{9 3 . 3 3}$ & 92.00 & 92.67 & 91.33 & 92.00 \\
Led & 67.60 & 68.20 & 67.00 & 67.00 & 67.00 & $\mathbf{6 8 . 4 0}$ \\
Mag & 79.49 & 79.18 & 77.71 & 77.28 & 76.97 & $\mathbf{8 0 . 1 3}$ \\
New & 91.63 & 90.70 & 92.09 & $\mathbf{9 2 . 5 6}$ & 91.63 & 91.16 \\
Pag & 94.34 & $\mathbf{9 5 . 2 5}$ & 94.16 & 94.34 & 94.16 & 94.34 \\
Pho & 81.98 & 81.92 & 79.03 & 79.70 & 79.87 & $\mathbf{8 2 . 7 2}$ \\
Pim & 72.65 & $\mathbf{7 5 . 0 0}$ & 73.05 & 72.65 & 73.18 & 73.56 \\
Rin & 81.89 & $\mathbf{8 6 . 7 6}$ & 75.54 & 78.11 & 77.97 & 86.49 \\
Sah & 69.89 & $\mathbf{7 0 . 9 9}$ & 68.18 & 68.61 & 69.03 & 69.25 \\
Sat & $\mathbf{7 9 . 8 4}$ & 78.54 & 77.29 & 78.38 & 76.98 & 79.00 \\
Seg & $\mathbf{9 2 . 2 1}$ & 91.56 & 90.00 & 90.69 & 90.39 & 92.03 \\
Tit & $\mathbf{7 8 . 8 7}$ & $\mathbf{7 8 . 8 7}$ & $\mathbf{7 8 . 8 7}$ & $\mathbf{7 8 . 8 7}$ & $\mathbf{7 8 . 8 7}$ & $\mathbf{7 8 . 8 7}$ \\
Two & 81.35 & 86.35 & 77.03 & 77.70 & 74.59 & $\mathbf{8 8 . 7 8}$ \\
Veh & 64.12 & $\mathbf{6 6 . 0 8}$ & 64.30 & 64.89 & 62.29 & 66.07 \\
Win & 93.25 & $\mathbf{9 4 . 3 7}$ & 94.35 & 88.73 & 91.59 & 94.37 \\
Wis & 95.17 & $\mathbf{9 6 . 3 4}$ & 95.46 & 95.32 & 94.88 & 96.05 \\
Yea & 56.47 & $\mathbf{5 7 . 6 8}$ & 53.91 & 53.97 & 55.12 & 56.13 \\
\hline Mean & 77.33 & 78.33 & 76.44 & 76.66 & 76.60 & $\mathbf{7 8 . 6 1}$ \\
\hline
\end{tabular}

Transactions on, vol. 18, no. 1, pp. 183-190, 1988.

[16] B. S. Ahn, "A priori identification of preferred alternatives of OWA operators by relational analysis of arguments," Information Sciences, vol. 180 , no. 23 , pp. $4572-4581,2010$.

[17] Y.-L. He, J. N. Liu, Y.-X. Hu, and X.-Z. Wang, "OWA operator based link prediction ensemble for social network," Expert Systems with Applications, vol. 42, no. 1, pp. 21 - 50, 2015.

[18] B. Llamazares, "Constructing choquet integral-based operators that generalize weighted means and OWA operators," Information Fusion, vol. 23 , pp. $131-138,2015$.

[19] J. M. Merigó and A. M. Gil-Lafuente, "Decision-making in sport management based on the OWA operator," Expert Systems with Applications, vol. 38 , no. 8, pp. 10408 - 10413, 2011.

[20] X. Sang and X. Liu, "An analytic approach to obtain the least square deviation OWA operator weights," Fuzzy Sets and Systems, vol. 240, pp. $103-116,2014$.

[21] H. Bustince, P. Burillo, and F. Soria, "Automorphisms, negations and implication operators," Fuzzy Sets and Systems, vol. 134, no. 2, pp. 209-229, 2013.

[22] H. Bustince, M. Pagola, and E. Barrenechea, "Construction of fuzzy indices from fuzzy DI-subsethood measures: Application to the global comparison of images," Information Sciences, vol. 177, no. 3, pp. 906 $-929,2007$.
TABLE XIII: Detailed results in testing using the power measure genetically adjusted (Power_GA.

\begin{tabular}{ccccccc}
\hline Dataset & Product & Minimum & Lukasiewicz & Drastic & Nilpotent & Hamacher \\
\hline App & 80.13 & 81.17 & 81.17 & 83.03 & $\mathbf{8 3 . 9 8}$ & 82.99 \\
Bal & 82.40 & $\mathbf{8 2 . 7 2}$ & 80.32 & 76.80 & 81.28 & 82.72 \\
Ban & $\mathbf{8 6 . 3 2}$ & 85.28 & 84.40 & 82.72 & 84.21 & 85.96 \\
Bnd & 64.00 & 70.25 & 71.02 & 65.56 & 69.37 & $\mathbf{7 2 . 1 3}$ \\
Bup & $\mathbf{6 6 . 9 6}$ & 61.16 & 65.22 & 63.48 & 64.06 & 65.80 \\
Cle & 55.58 & 56.26 & 55.20 & $\mathbf{5 6 . 8 9}$ & 54.54 & 55.58 \\
Eco & 76.51 & 78.57 & 74.72 & 75.61 & 77.41 & $\mathbf{8 0 . 0 7}$ \\
Gal & 64.02 & $\mathbf{6 4 . 9 6}$ & 64.49 & 62.17 & 64.51 & 63.10 \\
Hab & 72.52 & 71.87 & 73.18 & 73.20 & $\mathbf{7 3 . 8 4}$ & 72.21 \\
Hay & $\mathbf{7 9 . 4 9}$ & 77.95 & 77.98 & 78.77 & 78.75 & $\mathbf{7 9 . 4 9}$ \\
Iri & 91.33 & 92.67 & 92.00 & 92.67 & $\mathbf{9 4 . 0 0}$ & 93.33 \\
Led & 68.20 & $\mathbf{6 8 . 8 0}$ & 68.20 & 67.00 & 68.40 & 68.60 \\
Mag & 78.86 & $\mathbf{8 0 . 2 3}$ & 79.39 & 77.28 & 79.55 & 79.76 \\
New & 94.88 & 93.95 & 93.49 & 92.56 & 93.95 & $\mathbf{9 5 . 3 5}$ \\
Pag & 94.16 & 94.16 & 94.70 & 94.34 & $\mathbf{9 4 . 8 9}$ & 94.34 \\
Pho & 82.98 & 82.61 & 81.25 & 79.70 & 81.11 & $\mathbf{8 3 . 8 3}$ \\
Pim & 74.60 & $\mathbf{7 6 . 0 4}$ & 74.09 & 72.65 & 74.47 & 73.44 \\
Rin & $\mathbf{9 0 . 9 5}$ & 90.27 & 88.65 & 78.11 & 89.19 & 88.78 \\
Sah & 68.82 & $\mathbf{7 1 . 6 5}$ & 70.56 & 68.61 & 70.55 & 70.77 \\
Sat & 79.84 & 79.47 & 78.07 & 78.38 & 79.63 & $\mathbf{8 0 . 4 0}$ \\
Seg & $\mathbf{9 3 . 4 6}$ & 92.42 & 90.74 & 90.69 & 90.39 & 93.33 \\
Tit & $\mathbf{7 8 . 8 7}$ & $\mathbf{7 8 . 8 7}$ & $\mathbf{7 8 . 8 7}$ & $\mathbf{7 8 . 8 7}$ & $\mathbf{7 8 . 8 7}$ & $\mathbf{7 8 . 8 7}$ \\
Two & 84.46 & 84.86 & 83.78 & 77.70 & 85.00 & $\mathbf{8 5 . 2 7}$ \\
Veh & 64.71 & $\mathbf{6 8 . 4 4}$ & 62.53 & 64.89 & 64.90 & 68.20 \\
Win & 93.79 & 95.51 & 93.78 & 88.73 & $\mathbf{9 7 . 1 6}$ & 96.63 \\
Wis & $\mathbf{9 7 . 2 2}$ & 96.63 & 95.76 & 95.32 & 96.63 & 96.78 \\
Yea & 55.73 & 56.33 & 56.33 & 53.97 & 56.47 & $\mathbf{5 6 . 5 3}$ \\
\hline Mean & 78.55 & 79.00 & 78.14 & 76.66 & 78.78 & $\mathbf{7 9 . 4 2}$ \\
\hline
\end{tabular}

[23] H. Bustince, E. Barrenechea, and M. Pagola, "Image thresholding using restricted equivalence functions and maximizing the measures of similarity," Fuzzy Sets and Systems, vol. 158, no. 5, pp. 496 - 516, 2007.

[24] E. Barrenechea, H. Bustince, J. Fernandez, D. Paternain, and J. A. Sanz, "Using the Choquet integral in the fuzzy reasoning method of fuzzy rule-based classification systems," Axioms, vol. 2, no. 2, pp. 208-223, 2013.

[25] H. Ishibuchi, T. Nakashima, and M. Nii, Classification and modeling with linguistic information granules: Advanced approaches to linguistic Data Mining. Springer-Verlag, Berlin, 2004.

[26] _- Classification and Modeling with Linguistic Information Granules, Advanced Approaches to Linguistic Data Mining, ser. Advanced Information Processing. Berlin: Springer, 2005.

[27] H. Ishibuchi and Y. Nojima, "Pattern classification with linguistic rules," in Fuzzy Sets and Their Extensions: Representation, Aggregation and Models, ser. Studies in Fuzziness and Soft Computing, H. Bustince, F. Herrera, and J. Montero, Eds. Berlin: Springer, 2008, vol. 220, pp. 377-395.

[28] O. Cordon, M. J. del Jesus, and F. Herrera, "A proposal on reasoning methods in fuzzy rule-based classification systems," International Journal of Approximate Reasoning, vol. 20, no. 1, pp. 21 - 45, 1999.

[29] M. Elkano, M. Galar, J. Sanz, A. Fernandez, E. Barrenechea, F. Herrera, and H. Bustince Sola, "Enhancing multi-class classification in farchd fuzzy classifier: On the synergy between n-dimensional overlap functions and decomposition strategies," IEEE Transactions on Fuzzy Systems, 2014, in press.

[30] S. R. Samantaray, K. El-Arroudi, G. Joos, and I. Kamwa, "A fuzzy rulebased approach for islanding detection in distributed generation," Power Delivery, IEEE Transactions on, vol. 25, no. 3, pp. 1427-1433, 2010.

[31] J. A. Sanz, M. Galar, A. Jurio, A. Brugos, M. Pagola, and H. Bustince, "Medical diagnosis of cardiovascular diseases using an interval-valued fuzzy rule-based classification system," Applied Soft Computing, vol. 20, pp. 103 - 111, 2014.

[32] J. Sanz, A. Fernández, H. Bustince, and F. Herrera, "A genetic tuning to improve the performance of fuzzy rule-based classification systems with interval-valued fuzzy sets: Degree of ignorance and lateral position," International Journal of Approximate Reasoning, vol. 52, no. 6, pp. 751-766, 2011.

[33] J. A. Sanz, A. Fernández, H. Bustince, and F. Herrera, "Improving the performance of fuzzy rule-based classification systems with intervalvalued fuzzy sets and genetic amplitude tuning," Information Sciences, vol. 180 , no. 19 , pp. $3674-3685,2010$.

[34] H. Ishibuchi and T. Nakashima, "Effect of rule weights in fuzzy rulebased classification systems," Fuzzy Systems, IEEE Transactions on, vol. 9, no. 4, pp. 506-515, 2001.

[35] L. J. Eshelman, "The CHC adaptive search algorithm: How to have safe search when engaging in nontraditional genetic recombination," in Foundations of Genetic Algorithms, G. J. E. Rawlings, Ed. San Francisco: Morgan Kaufmann, 1991, pp. 265-283. 
[36] J. Alcalá-Fdez, A. Fernández, J. Luengo, J. Derrac, S. García, L. Sánchez, and F. Herrera, "KEEL data-mining software tool: Data set repository, integration of algorithms and experimental analysis framework," Journal of Multiple-Valued Logic and Soft Computing, vol. 17, no. 2-3, pp. 255-287, 2011.

[37] G. Lucca, J. Sanz, R. Vargas, G. P. Dimuro, B. Bedregal, and H. Bustince, "Using t-norms for generalizing Choquet integrals with an application to fuzzy rule-based classification systems," in Recent Advances on Fuzzy Systems, Proceedings of III Brazilian Congress on Fuzzy Systems (III CBSF), B. Bedregal, F. Gomide, F. Bergamaschi, L. Barros, R. Santiago, R. Moares, and W. Seixas, Eds. Joao Pessoa: SBMAC, 2014, pp. $300-3102$.

[38] J. Sanz, A. Fernández, H. Bustince, and F. Herrera, "IVTURS: A linguistic fuzzy rule-based classification system based on a new intervalvalued fuzzy reasoning method with tuning and rule selection," IEEE Transactions on Fuzzy Systems, vol. 21, no. 3, pp. 399-411, 2013.

[39] M. Galar, A. Fernández, E. Barrenechea, H. Bustince, and F. Herrera, "An overview of ensemble methods for binary classifiers in multi-class problems: Experimental study on one-vs-one and one-vs-all schemes," Pattern Recognition, vol. 44, no. 8, pp. 1761 - 1776, 2011.

[40] — "A review on ensembles for the class imbalance problem: Bagging-, boosting-, and hybrid-based approaches," Systems, Man, and Cybernetics, Part C: Applications and Reviews, IEEE Transactions on, vol. 42, no. 4, pp. 463-484, 2012.

[41] S. García, A. Fernández, J. Luengo, and F. Herrera, "A study of statistical techniques and performance measures for genetics-based machine learning: Accuracy and interpretability," Soft Computing, vol. 13, no. 10, pp. 959-977, 2009

[42] D. Sheskin, Handbook of parametric and nonparametric statistical procedures, 2nd ed. Chapman \& Hall/CRC, 2006.

[43] J. Demšar, "Statistical comparisons of classifiers over multiple data sets," Journal of Machine Learning Research, vol. 7, pp. 1-30, 2006.

[44] J. L. Hodges and E. L. Lehmann, "Ranks methods for combination of independent experiments in analysis of variance," Annals of Mathematical Statistics, vol. 33, pp. 482-497, 1962.

[45] S. Holm, "A simple sequentially rejective multiple test procedure," Scandinavian Journal of Statistics, vol. 6, pp. 65-70, 1979.

[46] F. Wilcoxon, "Individual comparisons by ranking methods," Biometrics, vol. 1 , pp. 80-83, 1945.

[47] B. C. Bedregal, G. P. Dimuro, H. Bustince, and E. Barrenechea, "New results on overlap and grouping functions," Information Sciences, vol. 249, pp. 148-170, 2013.

[48] G. P. Dimuro and B. Bedregal, "Archimedean overlap functions: The ordinal sum and the cancellation, idempotency and limiting properties," Fuzzy Sets and Systems, vol. 252, pp. 39 - 54, 2014.

[49] G. P. Dimuro, B. Bedregal, and R. H. N. Santiago, "On $(G, N)$ implications derived from grouping functions," Information Sciences, vol. 279 , pp. $1-17,2014$.

[50] B. C. Bedregal, G. P. Dimuro, and R. H. S. Reiser, "An approach to interval-valued R-implications and automorphisms," in Proceedings of the Joint 2009 International Fuzzy Systems Association World Congress and 2009 European Society of Fuzzy Logic and Technology Conference, IFSA/EUSFLAT, J. P. Carvalho, D. Dubois, U. Kaymak, and J. M. da Costa Sousa, Eds., 2009, pp. 1-6.

[51] B. C. Bedregal, G. P. Dimuro, R. H. N. Santiago, and R. H. S. Reiser, "On interval fuzzy S-implications," Information Sciences, vol. 180, no. 8, pp. $1373-1389,2010$

[52] G. Dimuro, "On interval fuzzy numbers," in 2011 Workshop-School on Theoretical Computer Science, WEIT 2011. Los Alamitos: IEEE, 2011, pp. 3-8.

[53] G. P. Dimuro, B. C. Bedregal, R. H. N. Santiago, and R. H. S. Reiser, "Interval additive generators of interval t-norms and interval t-conorms," Information Sciences, vol. 181, no. 18, pp. 3898 - 3916, 2011.

[54] G. P. Dimuro, B. R. C. Bedregal, R. H. S. Reiser, and R. H. N. Santiago, "Interval additive generators of interval t-norms," in Proceedings of the 15th International Workshop on Logic, Language, Information and Computation, WoLLIC 2008, Edinburgh, ser. Lecture Notes in Artificial Intelligence, W. Hodges and R. de Queiroz, Eds. Berlin: Springer, 2008, no. 5110, pp. 123-135.

[55] H. Bustince, M. Galar, B. Bedregal, A. Kolesárová, and R. Mesiar, "A new approach to interval-valued Choquet integrals and the problem of ordering in interval-valued fuzzy set applications," IEEE Transactions on Fuzzy Systems, vol. 21, no. 6, pp. 1150-1162, 2013.
Giancarlo Lucca G. Lucca has obtained his undergraduate and master degrees at Universidade Federal do Rio Grande (FURG/Brazil). Currently, is a $\mathrm{PhD}$ student at Universidad Publica de Navarra, in Pamplona, under the advising of Prof. Humberto Bustince.

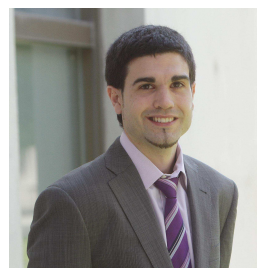

José Antonio Sanz José Antonio Sanz received the M.Sc. degree in computer sciences and the Ph.D. degree, both form the Public University of Navarre in 2008 and 2011 respectively. He is currently an Associate Lecturer with the Department of Automatics and Computation, Public University of Navarre. He is the author of 16 published original articles in international journals and is involved in teaching artificial intelligence for students of computer sciences. He received the best paper award in the FLINS 2012 international conference and the Pepe Millá award in 2014. His research interests include fuzzy techniques for classification problems, interval-valued fuzzy sets, genetic fuzzy systems, data mining and medical applications of soft computing techniques. Dr. Sanz is member of the European Society for Fuzzy Logic and Technology.

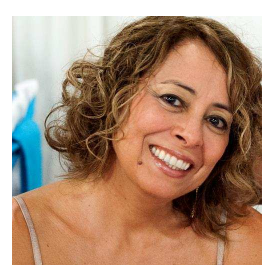

Graçaliz Pereira Dimuro Graçaliz Pereira Dimuro has obtained his undergraduate degree at Universidade Catlica de Pelotas (Brazil) and MSc and PhD degrees at the Instituto de Informtica of Universidade Federal do Rio Grande do Sul - UFRGS (Brazil). Currently, she is an Adjunct Professor at the Centro de Cincias Computacionais of Universidade Federal do Rio Grande - FURG (Brazil), researcher of the Brazilian research funding agency CNPQ and is in a sabbatic research year at Universidad Publica Borders Program (CNPq/Brazil). de Navarra, with a grant by the Science Without

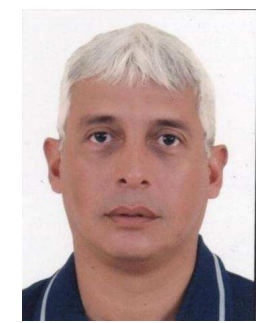

Benjamn Bedregal was born in Arica, Chile. He received the Engineering degree in Computing from the Tarapac University (UTA), in Arica, Chile, the M.Sc. degree in informatics and the Ph.D. degree in computer sciences from the Federal University of Pernambuco (UFPE), Recife, Brazil, in 1987 and 1996, respectively. In 1996, he became Assistant Professor at the Department of Informatics and Applied Mathematics, Federal University of Rio Grande do Norte (UFRN), Natal, Brazil, where he is currently a Full Professor. His research interests include: nonstandard fuzzy sets theory, aggregation functions, fuzzy connectives, clustering, fuzzy lattices, and fuzzy automata. 
Radko Mesiar is head of the Department of Mathematics at Faculty of Civil Engineering, STU Bratislava. Graduated at Comenius University, Faculty of Mathematics and Physics, in 1974, PhD at the same faculty obtained in 1979 with PhD thesis 'Subadditive martingale processes'. Since 1978 member of the Department of Mathematics at Faculty of Civil Engineering, STU Bratislava. DSc since 1996 (in Czech Republic, Academy of Sciences). Associate professor since 1983, full professor since 1998. Fellow member of Czech Academy of Sciences, Institute of Information and Automation, Praha (Czech Republic, since 1995) and of IRAFM, University of Ostrava (Czech Republic, since 2006). Co-author of two scientific monographs (Triangular Norms, Kluwer, 2000; Aggregation Functions, Cambridge University Press, 2009), 5 edited volumes and author of more than 200 papers in WoS journals. Founder and organizer of conferences FSTA and AGOP.

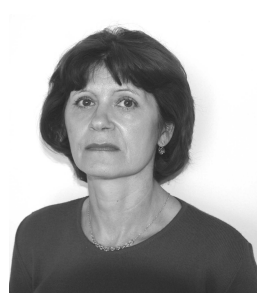

Anna Kolesárová comes from Slovakia. She received her M.Sc. degree in mathematics and physics from the Comenius University in Bratislava and $\mathrm{PhD}$. degree from the Slovak Academy of Sciences. In 2008 she became a full professor at the Institute of Information Engineering, Automation and Mathematics, Slovak University of Technology in Bratislava. Her current research interests include aggregation functions, with a special stress to copulas, measures and integrals, decision making and fuzzy arithmetic.

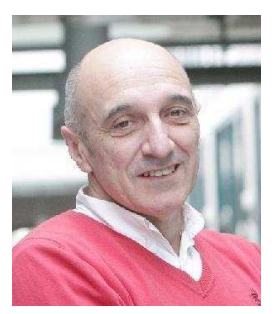

Humberto Bustince (M?08) received the Ph.D. degree in mathematics from the Public University of Navarra, Pamplona, Spain, in 1994. He is currently a Full Professor with the Department of Automatics and Computation, Public University of Navarra. He is the author of more than 65 published original articles and is involved in teaching artificial intelligence for students of computer sciences. His research interests include fuzzy logic theory, extensions of fuzzy sets (type-2 fuzzy sets, intervalvalued fuzzy sets, Atanassovs intuitionistic fuzzy sets), fuzzy measures, aggregation functions, and fuzzy techniques for image processing. Dr. Bustince is a board member of the European Society for Fuzzy Logic and Technology. He is the Editor-in-Chief of the Mathware and Soft Computing Magazine. 